\title{
Frequency-Domain Subsystem Identification with Application to Modeling Human Control Behavior
}

\author{
Xingye Zhang and Jesse B. Hoagg* \\ Department of Mechanical Engineering, University of Kentucky, Lexington, KY 40506-0503
}

\begin{abstract}
We present a frequency-domain subsystem identification algorithm that identifies unknown feedback and feedforward subsystems that are interconnected with a known subsystem. This method requires only accessible input and output measurements, applies to linear timeinvariant subsystems, and uses a candidate-pool approach to ensure asymptotic stability of the identified closed-loop transfer function. We analyze the algorithm in the cases of noiseless and noisy data. The main analytic result of this paper shows that the coefficients of the identified feedback and feedforward transfer functions are arbitrarily close to the true coefficients if the data noise is sufficiently small and the candidate pool is sufficiently dense. This subsystem identification approach has application to modeling the control behavior of humans interacting with and receiving feedback from a dynamic system. We apply the algorithm to data from a human-in-the-loop experiment to model a human's control behavior.
\end{abstract}

Keywords: subsystem identification; human-in-the-loop; human motor control

\section{Introduction}

Humans learn to interact with many complex dynamic systems. For example, humans learn to drive cars, fly planes, and ride unicycles. Moreover, humans learn to control all of these systems with virtually no a priori information. The control strategies that humans learn and the approaches used to learn them are currently unknown. The internal model hypothesis proposes that humans construct models of their body and the physical world, and these models are used for control [1, 2]. Although studies (e.g., [3-10]) provide evidence in support of the internal model hypothesis, this existing evidence is not conclusive [11].

Consider a scenario where a human interacts with a dynamic system by using feedback $y_{\mathrm{t}}$ and external information $r_{\mathrm{t}}$ (e.g., a command) to generate a control $u_{\mathrm{t}}$ as shown in Figure 1. In this scenario, the human is an unknown subsystem, which can include both feedback and feedforward. Modeling the human's control strategy can be viewed as a subsystem identification (SSID) problem, where $r_{\mathrm{t}}$ and $y_{\mathrm{t}}$ are measured and the dynamic system with which the human interacts is assumed to be known. The internal signals that the human uses to construct $u_{\mathrm{t}}$ are inaccessible (i.e., unmeasurable). For example, if $u_{\mathrm{t}}$ is the sum of feedback and feedforward terms, then these individual terms are inaccessible.

Existing methods for SSID are given in [12-24]. Specifically, [12-14] present methods for static subsystems, while [15-24] present methods for dynamic subsystems. In the dynamic SSID literature, the approaches in [15-19] are restricted to open-loop SSID, that is, identification of subsystems interconnected without feedback. We note that $[16,19]$ use open-loop SSID to model the dynamics of human subsystems. Specifically, [16] identifies a transfer

\footnotetext{
*Corresponding author; email: jhoagg@engr.uky.edu 


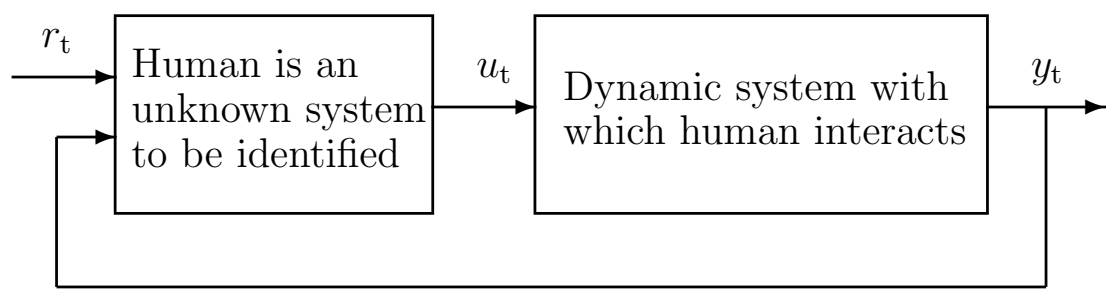

Figure 1: Modeling a human's control strategy can be viewed as an SSID problem, where $r_{\mathrm{t}}$ and $y_{\mathrm{t}}$ are measured and the dynamic system with which the human interacts is assumed to be known.

function that models a human's precision grip force dynamics, whereas [19] identifies two transfer functions that together model a human's oculomotor subsystem.

In contrast to [12-19], we focus on dynamic closed-loop SSID, that is, identification of dynamic subsystems with feedback. Existing dynamic closed-loop SSID methods include [2024]. In particular, [20] identifies a transfer function that models the behavior of a human subject interacting in feedback with a mechanical system. However, the method in [20] applies to systems with feedback only, that is, systems without feedforward. We note that the methods in [20-24] are time-domain techniques and yield identified models that may not result in an asymptotically stable closed-loop system.

This paper presents a new closed-loop SSID technique that: i) identifies feedback and feedforward subsystems, and ii) ensures asymptotic stability of the identified closed-loop transfer function. A closed-loop SSID method that addresses both i) and ii) is a new contribution of this paper. The method relies on a candidate-pool approach to accomplish ii). Another contribution of this paper is an analysis of the properties of the SSID algorithm in the cases of noiseless and noisy data. Our main analytic result shows that the coefficients of the identified feedback and feedforward transfer functions are arbitrarily close to the true coefficients if the data noise is sufficiently small and the candidate pool is sufficiently dense.

Characteristics i) and ii) of the SSID algorithm are motivated by the application to modeling human control behavior. First, humans generally use both anticipatory (feedforward) and reactive (feedback) control [1,2], which motivates i). Second, if a human-in-the-loop system has a bounded output, then it is desirable to identify subsystems that result in an asymptotically stable closed-loop transfer function, thus motivating ii). In addition, human control behavior is band limited; specifically, humans cannot produce motion with arbitrarily high frequency. Thus, we are interested in identifying models over a limited frequency range, which motivates the development of a new SSID technique in the frequency domain.

The frequency-domain SSID technique in this paper identifies unknown feedback and feedforward subsystems that are interconnected with a known subsystem, where all internal signals are assumed to be inaccessible, as shown in Figure 2. We present numerical examples to demonstrate the properties of the SSID algorithm. We also apply the SSID algorithm to data from an experiment designed to model the control behavior of a human subject interacting with a dynamic system. System identification approaches are also used in [25-31] to model human control behavior. However, [25-31] do not model both feedback and feedforward subsystems and do not ensure stability of the identified closed-loop model. A preliminary version of the SSID algorithm in this paper appeared in the conference proceedings [32]. However, this paper goes beyond the work of [32] by analyzing the properties of the SSID algorithm. 


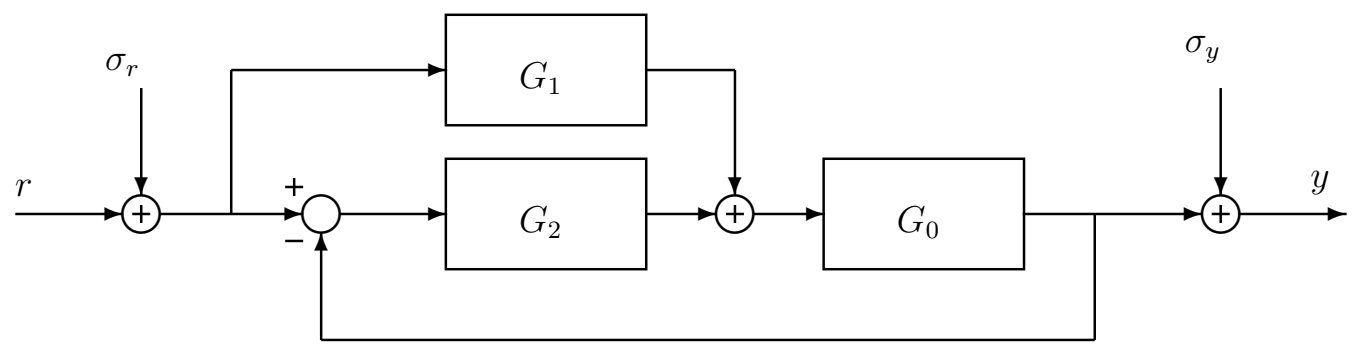

Figure 2: The input $r$ and output $y$ of this linear time-invariant system are measured, but all internal signals are inaccessible.

\section{Problem Formulation}

Consider the linear time-invariant system shown in Figure 2, where $r, y, \sigma_{r}$, and $\sigma_{y}$ are the Laplace transforms of the input, output, input noise, and output noise, respectively, and for $i=0,1,2, G_{i}: \mathbb{C} \rightarrow \mathbb{C}$ is a real rational transfer function. If $\sigma_{r}=0$ and $\sigma_{y}=0$, then the closed-loop transfer function from $r$ to $y$ is given by

$$
\tilde{G}(s) \triangleq \frac{G_{0}(s) G_{1}(s)+G_{0}(s) G_{2}(s)}{1+G_{0}(s) G_{2}(s)} .
$$

Next, let $N$ be a positive integer, and define $\mathcal{N} \triangleq\{1,2, \ldots, N\}$. For all $k \in \mathcal{N}$, let $\omega_{k} \in(0, \infty)$, where $\omega_{1}<\cdots<\omega_{N}$. Furthermore, for all $k \in \mathcal{N}$, define the closed-loop frequency response data

$$
H\left(\omega_{k}\right) \triangleq \frac{y\left(\jmath \omega_{k}\right)}{r\left(\jmath \omega_{k}\right)}=\tilde{G}\left(\jmath \omega_{k}\right)+\sigma\left(\jmath \omega_{k}\right)
$$

where $\sigma(s) \triangleq\left[\tilde{G}(s) \sigma_{r}(s)+\sigma_{y}(s)\right] / r(s)$. If $\sigma\left(\jmath \omega_{k}\right) \equiv 0$, then $\left\{H\left(\omega_{k}\right)\right\}_{k=1}^{N}$ is noiseless. In contrast, if $\sigma\left(\jmath \omega_{k}\right) \not \equiv 0$, then $\left\{H\left(\omega_{k}\right)\right\}_{k=1}^{N}$ is noisy.

We present a method to identify $G_{1}$ and $G_{2}$ provided that $G_{0}$ and $\left\{H\left(\omega_{k}\right)\right\}_{k=1}^{N}$ are known and $G_{0} \neq 0$. In this case, the closed-loop frequency response data $\left\{H\left(\omega_{k}\right)\right\}_{k=1}^{N}$ can be obtained from the accessible signals $r$ and $y$ and does not depend on the internal signals, which are not assumed to be measured.

For $i=0,1,2, G_{i}$ can be expressed as $G_{i}(s)=N_{i}(s) / D_{i}(s)$, where $N_{i}$ and $D_{i}$ are coprime, and $D_{i}$ is monic. The degrees of $N_{i}$ and $D_{i}$ are denoted by $n_{i} \triangleq \operatorname{deg} N_{i}$ and $d_{i} \triangleq \operatorname{deg} D_{i}$. Thus, (1) can be expressed as

$$
\tilde{G}(s)=\frac{N_{0}(s)\left[D_{2}(s) N_{1}(s)+D_{1}(s) N_{2}(s)\right]}{D_{1}(s)\left[D_{0}(s) D_{2}(s)+N_{0}(s) N_{2}(s)\right]} .
$$

We make the following assumptions:

(A1) $d_{1}, d_{2}, n_{1}$, and $n_{2}$ are known.

(A2) $d_{0}+d_{2}>n_{0}+n_{2}$.

(A3) $N>d_{0}+d_{1}+d_{2}+n_{0}+\max \left\{n_{1}+d_{2}, n_{2}+d_{1}\right\}$.

(A4) If $\lambda \in \mathbb{C}$ and $D_{1}(\lambda)\left[D_{0}(\lambda) D_{2}(\lambda)+N_{0}(\lambda) N_{2}(\lambda)\right]=0$, then $\operatorname{Re} \lambda<0$.

Assumption (A1) can be replaced by the assumption that upper bounds on $d_{1}, d_{2}, n_{1}$, and $n_{2}$ are known. However, we invoke (A1) for clarity of the presentation. Assumption (A2) states that the loop transfer function $G_{0} G_{2}$ is strictly proper. Assumption (A3) implies that the number $N$ of frequency response data points is sufficiently large. This assumption ensures that the minimization problem solved in the SSID has a unique solution. Assumption 
(A4) implies that $\tilde{G}$ is asymptotically stable, that is, the poles of $\tilde{G}$ are in the open-left-half complex plane.

Define $d \triangleq d_{1}+d_{2}+n_{2}+1$, and for all nonnegative integers $j$, let $\Gamma_{j}: \mathbb{C} \rightarrow \mathbb{C}^{j+1}$ be given by $\Gamma_{j}(s) \triangleq\left[\begin{array}{lllll}s^{j} & s^{j-1} & \cdots & s & 1\end{array}\right]^{\mathrm{T}}$. Consider the functions $\mathcal{N}_{1}: \mathbb{C} \times \mathbb{R}^{n_{1}+1} \rightarrow \mathbb{C}$ and $\mathcal{D}_{1}, \mathcal{N}_{2}, \mathcal{D}_{2}: \mathbb{C} \times \mathbb{R}^{d} \rightarrow \mathbb{C}$ given by

$$
\begin{array}{ll}
\mathcal{N}_{1}(s, \beta) \triangleq \Gamma_{n_{1}}^{\mathrm{T}}(s) \beta, & \mathcal{D}_{1}(s, \phi) \triangleq s^{d_{1}}+\Gamma_{d_{1}-1}^{\mathrm{T}}(s) E_{1} \phi, \\
\mathcal{N}_{2}(s, \phi) \triangleq \Gamma_{n_{2}}^{\mathrm{T}}(s) E_{2} \phi, & \mathcal{D}_{2}(s, \phi) \triangleq s^{d_{2}}+\Gamma_{d_{2}-1}^{\mathrm{T}}(s) E_{3} \phi,
\end{array}
$$

where $E_{1} \triangleq\left[\begin{array}{ll}I_{d_{1}} & 0_{d_{1} \times\left(d_{2}+n_{2}+1\right)}\end{array}\right] \in \mathbb{R}^{d_{1} \times d}, E_{2} \triangleq\left[\begin{array}{lll}0_{\left(n_{2}+1\right) \times d_{1}} & I_{n_{2}+1} & 0_{\left(n_{2}+1\right) \times d_{2}}\end{array}\right] \in \mathbb{R}^{\left(n_{2}+1\right) \times d}$, $E_{3} \triangleq\left[\begin{array}{ll}0_{d_{2} \times\left(d_{1}+n_{2}+1\right)} & I_{d_{2}}\end{array}\right] \in \mathbb{R}^{d_{2} \times d}, \beta \in \mathbb{R}^{n_{1}+1}$, and $\phi \in \mathbb{R}^{d}$. Next, consider the functions $\mathcal{G}_{1}: \mathbb{C} \times \mathbb{R}^{n_{1}+1} \times \mathbb{R}^{d} \rightarrow \mathbb{C}$ and $\mathcal{G}_{2}: \mathbb{C} \times \mathbb{R}^{d} \rightarrow \mathbb{C}$ given by

$$
\mathcal{G}_{1}(s, \beta, \phi) \triangleq \frac{\mathcal{N}_{1}(s, \beta)}{\mathcal{D}_{1}(s, \phi)}, \quad \mathcal{G}_{2}(s, \phi) \triangleq \frac{\mathcal{N}_{2}(s, \phi)}{\mathcal{D}_{2}(s, \phi)},
$$

which, for each $\beta \in \mathbb{R}^{n_{1}+1}$ and $\phi \in \mathbb{R}^{d}$, are real rational transfer functions.

Our objective is to determine $\beta$ and $\phi$ such that $\mathcal{G}_{1}$ and $\mathcal{G}_{2}$ approximate $G_{1}$ and $G_{2}$, respectively. To achieve this objective, consider the cost function

$$
J(\beta, \phi) \triangleq \sum_{k=1}^{N}\left|\frac{N_{0}\left(\jmath \omega_{k}\right)\left[\mathcal{D}_{2}\left(\jmath \omega_{k}, \phi\right) \mathcal{N}_{1}\left(\jmath \omega_{k}, \beta\right)+\mathcal{D}_{1}\left(\jmath \omega_{k}, \phi\right) \mathcal{N}_{2}\left(\jmath \omega_{k}, \phi\right)\right]}{\mathcal{D}_{1}\left(\jmath \omega_{k}, \phi\right)\left[D_{0}\left(\jmath \omega_{k}\right) \mathcal{D}_{2}\left(\jmath \omega_{k}, \phi\right)+N_{0}\left(\jmath \omega_{k}\right) \mathcal{N}_{2}\left(\jmath \omega_{k}, \phi\right)\right]}-H\left(\omega_{k}\right)\right|^{2},
$$

which is a measure of the difference between the frequency response data $\left\{H\left(\omega_{k}\right)\right\}_{k=1}^{N}$ and the closed-loop transfer function obtained from the estimates $\mathcal{G}_{1}$ and $\mathcal{G}_{2}$. Note that $J$ is nonlinear and nonconvex in $(\beta, \phi)$.

Let $\beta_{*} \in \mathbb{R}^{n_{1}+1}$ and $\phi_{*} \in \mathbb{R}^{d}$ be such that, for all $s \in \mathbb{C}, N_{1}(s)=\mathcal{N}_{1}\left(s, \beta_{*}\right), D_{1}(s)=$ $\mathcal{D}_{1}\left(s, \phi_{*}\right), N_{2}(s)=\mathcal{N}_{2}\left(s, \phi_{*}\right)$, and $D_{2}(s)=\mathcal{D}_{2}\left(s, \phi_{*}\right)$. If $\sigma\left(\jmath \omega_{k}\right) \equiv 0$, then $J\left(\beta_{*}, \phi_{*}\right)=0$, which implies that the true parameters $\beta_{*}$ and $\phi_{*}$ minimize the cost function (3) if the closed-loop frequency response data $\left\{H\left(\omega_{k}\right)\right\}_{k=1}^{N}$ is noiseless.

\section{Subsystem Identification}

We now develop an SSID algorithm to obtain estimates of $\beta_{*}$ and $\phi_{*}$, and thus estimates of $G_{1}$ and $G_{2}$. Consider the function $\tilde{\mathcal{G}}: \mathbb{C} \times \mathbb{R}^{n_{1}+1} \times \mathbb{R}^{d} \rightarrow \mathbb{C}$ defined by

$$
\tilde{\mathcal{G}}(s, \beta, \phi) \triangleq \frac{\tilde{\mathcal{N}}_{1}(s, \phi) \beta+\tilde{\mathcal{N}}_{2}(s, \phi)}{\tilde{\mathcal{D}}(s, \phi)}
$$

where

$$
\begin{aligned}
\tilde{\mathcal{D}}(s, \phi) & \triangleq \mathcal{D}_{1}(s, \phi)\left[D_{0}(s) \mathcal{D}_{2}(s, \phi)+N_{0}(s) \mathcal{N}_{2}(s, \phi)\right], \\
\tilde{\mathcal{N}}_{1}(s, \phi) & \triangleq N_{0}(s) \mathcal{D}_{2}(s, \phi) \Gamma_{n_{1}}^{\mathrm{T}}(s), \\
\tilde{\mathcal{N}}_{2}(s, \phi) & \triangleq N_{0}(s) \mathcal{D}_{1}(s, \phi) \mathcal{N}_{2}(s, \phi) .
\end{aligned}
$$

Note that $\tilde{\mathcal{G}}(s, \beta, \phi)$ is the closed-loop transfer function obtained using $\beta$ and $\phi$. It follows from $(3)-(7)$ that

$$
J(\beta, \phi)=\sum_{k=1}^{N}\left|\tilde{\mathcal{G}}\left(\jmath \omega_{k}, \beta, \phi\right)-H\left(\omega_{k}\right)\right|^{2}
$$

Next, we define

$$
\Omega_{0}(\phi) \triangleq \sum_{k=1}^{N}\left|\frac{\tilde{\mathcal{N}}_{2}\left(\jmath \omega_{k}, \phi\right)}{\tilde{\mathcal{D}}\left(\jmath \omega_{k}, \phi\right)}-H\left(\omega_{k}\right)\right|^{2} \in \mathbb{R}
$$




$$
\begin{aligned}
& \Omega_{1}(\phi) \triangleq 2 \operatorname{Re} \sum_{k=1}^{N}\left[\frac{\tilde{\mathcal{N}}_{1}\left(\jmath \omega_{k}, \phi\right)}{\tilde{\mathcal{D}}\left(\jmath \omega_{k}, \phi\right)}\right]^{*}\left[\frac{\tilde{\mathcal{N}}_{2}\left(\jmath \omega_{k}, \phi\right)}{\tilde{\mathcal{D}}\left(\jmath \omega_{k}, \phi\right)}-H\left(\omega_{k}\right)\right] \in \mathbb{R}^{n_{1}+1}, \\
& \Omega_{2}(\phi) \triangleq \operatorname{Re} \sum_{k=1}^{N}\left[\frac{\tilde{\mathcal{N}}_{1}\left(\jmath \omega_{k}, \phi\right)}{\tilde{\mathcal{D}}\left(\jmath \omega_{k}, \phi\right)}\right]^{*}\left[\frac{\tilde{\mathcal{N}}_{1}\left(\jmath \omega_{k}, \phi\right)}{\tilde{\mathcal{D}}\left(\jmath \omega_{k}, \phi\right)}\right] \in \mathbb{R}^{\left(n_{1}+1\right) \times\left(n_{1}+1\right)},
\end{aligned}
$$

where $[\cdot]^{*}$ denotes the complex conjugate transpose. Thus, (8) can be expressed as

$$
J(\beta, \phi)=\beta^{\mathrm{T}} \Omega_{2}(\phi) \beta+\Omega_{1}^{\mathrm{T}}(\phi) \beta+\Omega_{0}(\phi),
$$

which is convex in $\beta$, because, for each $\phi \in \mathbb{R}^{d}, \Omega_{2}(\phi)$ is positive semidefinite.

Define the set of $\phi \in \mathbb{R}^{d}$ such that $\tilde{\mathcal{G}}(s, \beta, \phi)$ is asymptotically stable, which is given by $\mathcal{S} \triangleq\left\{\phi \in \mathbb{R}^{d}: \tilde{\mathcal{D}}(s, \phi)\right.$ is Hurwitz $\}$.

The following result provides sufficient conditions such that $\Omega_{2}(\phi)$ is positive definite. The proof is in Appendix A.

Proposition 1. Consider $\Omega_{2}$ given by (11), and assume (A1)-(A3) are satisfied. Let $\phi \in \mathcal{S}$. Then, $\Omega_{2}(\phi)$ is positive definite.

For each $\phi \in \mathcal{S}$, the following result provides the global minimizer of $J(\beta, \phi)$. The proof is in Appendix A.

Proposition 2. Consider $J$ given by (12), and assume (A1)-(A3) are satisfied. Let $\phi \in \mathcal{S}$, and let $\beta \in \mathbb{R}^{n_{1}+1} \backslash\left\{-\frac{1}{2} \Omega_{2}^{-1}(\phi) \Omega_{1}(\phi)\right\}$. Then,

$$
J\left(-\frac{1}{2} \Omega_{2}^{-1}(\phi) \Omega_{1}(\phi), \phi\right)=\Omega_{0}(\phi)-\frac{1}{4} \Omega_{1}^{\mathrm{T}}(\phi) \Omega_{2}^{-1}(\phi) \Omega_{1}(\phi)<J(\beta, \phi) .
$$

Next, let $M$ be a positive integer, and let $\Phi \subseteq \mathcal{S}$ be a set with $M$ elements. We call $\Phi$ the candidate pool. Define $\mathcal{M} \triangleq\{1,2, \ldots, M\}$. We now create a candidate sequence using the $M$ elements in the candidate pool $\Phi$. For $i, j \in \mathcal{M}$, let $\phi_{i}, \phi_{j} \in \Phi$ be such that if $i \neq j$, then $\phi_{i} \neq \phi_{j}$. The sequence $\left\{\phi_{i}\right\}_{i=1}^{M}$ is not unique; however, the order of the sequence is selected arbitrarily.

Now, for all $i \in \mathcal{M}$, define the quadratic cost function

$$
\left.\mathcal{J}_{i}(\beta) \triangleq J(\beta, \phi)\right|_{\phi=\phi_{i}}=\beta^{\mathrm{T}} \Omega_{2}\left(\phi_{i}\right) \beta+\Omega_{1}^{\mathrm{T}}\left(\phi_{i}\right) \beta+\Omega_{0}\left(\phi_{i}\right) .
$$

Since $\phi_{1}, \ldots, \phi_{M} \in \Phi \subseteq \mathcal{S}$, Proposition 1 implies that $\Omega_{2}\left(\phi_{1}\right), \ldots, \Omega_{2}\left(\phi_{M}\right)$ are positive definite. The following result is a consequence of Proposition 2 and provides the unique global minimizer of $\mathcal{J}_{i}$ for each $i \in \mathcal{M}$.

Proposition 3. Consider $\partial_{i}$ given by (13), assume (A1)-(A3) are satisfied, and assume $\Phi \subseteq \mathcal{S}$. Let $i \in \mathcal{M}$, and let $\beta \in \mathbb{R}^{n_{1}+1} \backslash\left\{-\frac{1}{2} \Omega_{2}^{-1}\left(\phi_{i}\right) \Omega_{1}\left(\phi_{i}\right)\right\}$. Then,

$$
\mathcal{J}_{i}\left(-\frac{1}{2} \Omega_{2}^{-1}\left(\phi_{i}\right) \Omega_{1}\left(\phi_{i}\right)\right)=\Omega_{0}\left(\phi_{i}\right)-\frac{1}{4} \Omega_{1}^{\mathrm{T}}\left(\phi_{i}\right) \Omega_{2}^{-1}\left(\phi_{i}\right) \Omega_{1}\left(\phi_{i}\right)<\mathcal{J}_{i}(\beta) .
$$

Proposition 3 implies that for each $i \in \mathcal{M}, \beta_{i} \triangleq-\frac{1}{2} \Omega_{2}^{-1}\left(\phi_{i}\right) \Omega_{1}\left(\phi_{i}\right)$ is the global minimizer of $\mathcal{J}_{i}$. Next, let $\ell \in \mathcal{M}$ be the smallest integer such that $\mathcal{J}_{\ell}\left(\beta_{\ell}\right)=\min _{i \in \mathcal{M}} \mathcal{J}_{i}\left(\beta_{i}\right)$. Then, the identified parameters are $\beta^{+} \triangleq \beta_{\ell}$ and $\phi^{+} \triangleq \phi_{\ell}$, and the identified transfer functions are $G_{1}^{+}(s) \triangleq \mathcal{G}_{1}\left(s, \beta^{+}, \phi^{+}\right)$and $G_{2}^{+}(s) \triangleq \mathcal{G}_{2}\left(s, \phi^{+}\right)$. Note that $\arg \min _{i \in \mathcal{M}} \mathcal{J}_{i}\left(\beta_{i}\right)$ is not necessarily unique. In this case, $\ell \in \mathcal{M}$ is the smallest integer such that $\mathcal{J}_{\ell}\left(\beta_{\ell}\right)=\min _{i \in \mathcal{M}} \mathcal{J}_{i}\left(\beta_{i}\right)$. However, in practice, $\arg \min _{i \in \mathcal{M}} \mathcal{J}_{i}\left(\beta_{i}\right)$ is generally unique. We now summarize this SSID method. 
Algorithm 1. Consider the closed-loop transfer function (1), where $G_{0}$ is known. Assume $\left\{H\left(\omega_{k}\right)\right\}_{k=1}^{N}$ is known, and (A1)-(A4) are satisfied. Then, the subsystem identification algorithm is as follows:

Step 1. Generate the candidate pool $\Phi \subseteq \mathcal{S}$ and candidate sequence $\left\{\phi_{i}\right\}_{i=1}^{M}$.

Step 2. For each $i \in \mathcal{M}$, find $\beta_{i} \triangleq-\frac{1}{2} \Omega_{2}^{-1}\left(\phi_{i}\right) \Omega_{1}\left(\phi_{i}\right)$, which is the global minimizer of $\mathcal{J}_{i}$.

Step 3. Find the smallest integer $\ell \in \mathcal{M}$ such that $\mathcal{J}_{\ell}\left(\beta_{\ell}\right)=\min _{i \in \mathcal{M}} \mathcal{J}_{i}\left(\beta_{i}\right)$.

Step 4. The identified parameters are $\beta^{+}=\beta_{\ell}$ and $\phi^{+}=\phi_{\ell}$.

Step 5. The identified transfer functions are $G_{1}^{+}(s)=\mathcal{G}_{1}\left(s, \beta^{+}, \phi^{+}\right)$and $G_{2}^{+}(s)=\mathcal{G}_{2}\left(s, \phi^{+}\right)$.

In the next two sections, we analyze Algorithm 1. Specifically, we analyze how the identified parameters $\beta^{+}$and $\phi^{+}$relate to the true parameters $\beta_{*}$ and $\phi_{*}$.

\section{Analysis with Noiseless Frequency Response Data}

In this section, we analyze Algorithm 1 under the assumption of noiseless frequency response data. We assume that for all $k \in \mathcal{N}, \sigma\left(\jmath \omega_{k}\right)=0$, which implies that for all $k \in \mathcal{N}$, $H\left(\omega_{k}\right)=\tilde{G}\left(\jmath \omega_{k}\right)$. The following result relates $\beta_{*}$ and $\phi_{*}$ if $\sigma\left(\jmath \omega_{k}\right) \equiv 0$. The proof is in Appendix B.

Proposition 4. Assume (A1)-(A4) are satisfied, and assume for all $k \in \mathcal{N}, \sigma\left(\jmath \omega_{k}\right)=0$. Then, $\beta_{*}=-\frac{1}{2} \Omega_{2}^{-1}\left(\phi_{*}\right) \Omega_{1}\left(\phi_{*}\right)$ and $J\left(\beta_{*}, \phi_{*}\right)=0$.

The following result provides sufficient conditions on $\beta \in \mathbb{R}^{n_{1}+1}$ and $\phi \in \mathcal{S}$ such that $\tilde{\mathcal{G}}(s, \beta, \phi) \equiv \tilde{G}(s)$. The proof is in Appendix B.

Proposition 5. Let $\beta \in \mathbb{R}^{n_{1}+1}$ and $\phi \in \mathbb{R}^{d}$. Assume (A3) is satisfied, and assume $\sum_{k=1}^{N}\left|\tilde{\mathcal{G}}\left(\jmath \omega_{k}, \beta, \phi\right)-\tilde{G}\left(\jmath \omega_{k}\right)\right|=0$. Then, for all $s \in \mathbb{C}, \tilde{\mathcal{G}}(s, \beta, \phi)=\tilde{G}(s)$.

Proposition 5 provides sufficient conditions such that $\tilde{\mathcal{G}}(s, \beta, \phi) \equiv \tilde{G}(s) \equiv \tilde{\mathcal{G}}\left(s, \beta_{*}, \phi_{*}\right)$; however, these conditions are not sufficient to conclude that $\left[\begin{array}{lll}\beta^{\mathrm{T}} & \phi^{\mathrm{T}}\end{array}\right]$ equals $\left[\begin{array}{cc}\beta_{*}^{\mathrm{T}} & \phi_{*}^{\mathrm{T}}\end{array}\right]$. The following example demonstrates this case.

Example 1. Consider the closed-loop transfer function (1), where $G_{0}(s)=1 /(s+2)$, $G_{1}(s)=(s+3) /(s+1)$, and $G_{2}(s)=1$. Note that $\beta_{*}=\left[\begin{array}{ll}1 & 3\end{array}\right]^{\mathrm{T}}, \phi_{*}=\left[\begin{array}{ll}1 & 1\end{array}\right]^{\mathrm{T}}$, and $\tilde{G}(s)=(2 s+4) /\left(s^{2}+4 s+3\right)$. Let $\beta=\left[\begin{array}{ll}3 & 7\end{array}\right]^{\mathrm{T}} \neq \beta_{*}$ and $\phi=\left[\begin{array}{ll}3 & -1\end{array}\right]^{\mathrm{T}} \neq \phi_{*}$, and it follows that $\tilde{\mathcal{G}}(s, \beta, \phi) \equiv \tilde{G}(s) \equiv \tilde{\mathcal{G}}\left(s, \beta_{*}, \phi_{*}\right)$.

Example 1 shows that there can exist $\beta \in \mathbb{R}^{n_{1}+1}$ and $\phi \in \mathcal{S}$ such that $\left[\beta^{\mathrm{T}} \phi^{\mathrm{T}}\right] \neq$ $\left[\begin{array}{cc}\beta_{*}^{\mathrm{T}} & \phi_{*}^{\mathrm{T}}\end{array}\right]$ and $\tilde{\mathcal{G}}(s, \beta, \phi) \equiv \tilde{\mathcal{G}}\left(s, \beta_{*}, \phi_{*}\right)$. In this case, the SSID problem is not well posed, because $J$ does not have a unique minimizer, and $\left(\beta_{*}, \phi_{*}\right)$ cannot be determined uniquely from the noiseless closed-loop frequency response data $\left\{H\left(\omega_{k}\right)\right\}_{k=1}^{N}$.

We now impose additional assumption to ensure that if $\tilde{\mathcal{G}}(s, \beta, \phi) \equiv \tilde{G}(s)$, then $\beta=\beta_{*}$ and $\phi=\phi_{*}$. First, let $\Psi \subseteq \mathbb{R}^{d}$ be a compact and perfect (i.e., closed with no isolated point) set containing $\phi_{*}$. Note that $\Psi$ can be selected sufficiently large to ensure $\phi_{*} \in \Psi$. We assume $\Psi$ is known. In practice, $\Psi$ is used to generate the candidate pool. For the remainder of this paper, we impose the assumption:

(A5) If $\beta \in \mathbb{R}^{n_{1}+1}, \phi \in \Psi \cap \mathcal{S}$, and $\tilde{\mathcal{G}}(s, \beta, \phi) \equiv \tilde{G}(s)$, then $\beta=\beta_{*}$ and $\phi=\phi_{*}$.

Assumption (A5) implies that $\left[\begin{array}{ll}\beta_{*}^{\mathrm{T}} & \phi_{*}^{\mathrm{T}}\end{array}\right]^{\mathrm{T}}$ is the only element in $\mathbb{R}^{n_{1}+1} \times(\Psi \cap \mathcal{S})$ that yields the closed-loop transfer function $\tilde{G}$. 


\section{1. $\phi_{*}$ in the candidate pool $\Phi$}

The following result provides sufficient conditions such that the identified transfer functions $G_{1}^{+}$and $G_{2}^{+}$are equal to $G_{1}$ and $G_{2}$, respectively.

Theorem 1. Consider the closed-loop transfer function (1), where $G_{0}$ is known. Assume $\left\{H\left(\omega_{k}\right)\right\}_{k=1}^{N}$ is known, (A1)-(A5) are satisfied, and for all $k \in \mathcal{N}, \sigma\left(\jmath \omega_{k}\right)=0$. Furthermore, consider Algorithm 1 with the candidate pool $\Phi \subseteq(\Psi \cap \mathcal{S})$, and assume $\phi_{*} \in \Phi$. Let $\beta^{+}$ and $\phi^{+}$denote the identified parameters obtained from Algorithm 1 . Then, $\beta^{+}=\beta_{*}$ and $\phi^{+}=\phi_{*}$.

Proof. Since $\phi_{*} \in \Phi$, it follows that there exists $m \in \mathcal{M}$ such that $\phi_{m}=\phi_{*}$. Next, since for all $k \in \mathcal{N}, \sigma\left(\jmath \omega_{k}\right)=0$, it follows from Proposition 3 and Proposition 4 that $\beta_{m}=-\frac{1}{2} \Omega_{2}^{-1}\left(\phi_{m}\right) \Omega_{1}\left(\phi_{m}\right)=-\frac{1}{2} \Omega_{2}^{-1}\left(\phi_{*}\right) \Omega_{1}\left(\phi_{*}\right)=\beta_{*}$. Thus, (13) and Proposition 4 imply that $\mathcal{J}_{m}\left(\beta_{m}\right)=J\left(\beta_{m}, \phi_{m}\right)=J\left(\beta_{*}, \phi_{*}\right)=0$.

Since for all $i \in \mathcal{M}, \mathcal{J}_{i}\left(\beta_{i}\right) \geq 0$ and $\mathcal{J}_{m}\left(\beta_{m}\right)=0$, it follows that $J\left(\beta^{+}, \phi^{+}\right)=\min _{i \in \mathcal{M}} \mathcal{J}_{i}\left(\beta_{i}\right)=$ $\partial_{m}\left(\beta_{m}\right)=0$. Since for all $k \in \mathcal{N}, H\left(\omega_{k}\right)=\tilde{G}\left(\jmath \omega_{k}\right)$, it follows from (8) that $0=J\left(\beta^{+}, \phi^{+}\right)=$ $\sum_{k=1}^{N}\left|\tilde{\mathcal{G}}\left(\jmath \omega_{k}, \beta^{+}, \phi^{+}\right)-\tilde{G}\left(\jmath \omega_{k}\right)\right|^{2}$. Thus, Proposition 5 implies that $\tilde{\mathcal{G}}\left(s, \beta^{+}, \phi^{+}\right) \equiv \tilde{G}(s)$. Since, in addition, $\phi^{+} \in \Phi \subseteq(\Psi \cap \mathcal{S})$, (A5) implies that $\beta^{+}=\beta_{*}$ and $\phi^{+}=\phi_{*}$.

\section{2. $\phi_{*}$ not necessarily in the candidate pool $\Phi$}

We now extend the analysis in the previous section to address the case where $\phi_{*}$ is not necessarily in the candidate pool. It follows from Proposition 1 that for all $\phi \in \mathcal{S}, \Omega_{2}(\phi)$ is positive definite, which implies that $\theta: \mathcal{S} \rightarrow \mathbb{R}^{n_{1}+1}$ given by

$$
\theta(\phi) \triangleq-\frac{1}{2} \Omega_{2}^{-1}(\phi) \Omega_{1}(\phi)
$$

is well defined on $\mathcal{S}$. Define $Q: \mathcal{S} \rightarrow[0, \infty)$ by

$$
Q(\phi) \triangleq J(\theta(\phi), \phi)
$$

The following result addresses continuity of $\theta$ and $Q$. The proof is in Appendix C.

Proposition 6. Assume (A1)-(A3) are satisfied. Then, $\theta$ and $Q$ are continuous on $\mathcal{S}$.

It follows from (A4) that $\tilde{\mathcal{D}}\left(s, \phi_{*}\right)$ is Hurwitz. Thus, there exists $\rho<0$ such that if $\lambda \in \mathbb{C}$ and $\tilde{\mathcal{D}}\left(\lambda, \phi_{*}\right)=0$, then $\operatorname{Re} \lambda<\rho$. We assume $\rho$ is known. Note that $\rho<0$ can be selected such that $|\rho|$ is arbitrarily small, which ensures that $\mathcal{S}_{\rho} \triangleq\left\{\phi \in \mathbb{R}^{d}: \tilde{\mathcal{D}}(s+\rho, \phi)\right.$ is Hurwitz $\}$ contains $\phi_{*}$. In practice, $\mathcal{S}_{\rho}$ is used to generate the candidate pool.

The following propositions are needed for the main result of this section. The proofs are in Appendix D. Note that $\overline{\Psi \cap \mathcal{S}_{\rho}}$ denotes the closure of $\Psi \cap \mathcal{S}_{\rho}$.

Proposition 7. $\Psi \cap \mathcal{S}_{\rho}$ is bounded and contains no isolated points.

Proposition 8. $\overline{\Psi \cap \mathcal{S}_{\rho}} \subseteq \mathcal{S}$ is compact.

Let $\mathbb{Z}^{+}$denote the set of positive integers, let $n \in \mathbb{Z}^{+}$, and define the open ball of radius $\epsilon>0$ centered at $c \in \mathbb{R}^{n}$ by $\mathbb{B}_{\epsilon}(c) \triangleq\left\{x \in \mathbb{R}^{n}:\|x-c\|<\epsilon\right\}$. We now define a convergent sequence of finite sets.

Definition 1. Let $n \in \mathbb{Z}^{+}$, and let $\Delta \subseteq \mathbb{R}^{n}$ be bounded and contain no isolated points. For all $j \in \mathbb{Z}^{+}$, let $\Delta_{j} \subseteq \Delta$ be a finite set. Then, $\left\{\Delta_{j}\right\}_{j=1}^{\infty}$ converges to $\Delta$ if for each $x \in \Delta$, there exists a sequence $\left\{x_{j}: x_{j} \in \Delta_{j}\right\}_{j=1}^{\infty}$ such that for all $\epsilon>0$, there exists $L \in \mathbb{Z}^{+}$such that for $j>L, x_{j} \in \mathbb{B}_{\epsilon}(x)$. 
In the following result, we consider Algorithm 1 with a sequence of candidate pools that converges to $\Psi \cap \mathcal{S}_{\rho}$. This result demonstrates that a sufficiently dense candidate pool yields identified parameters $\beta^{+}$and $\phi^{+}$that are arbitrarily close to $\beta_{*}$ and $\phi_{*}$.

Theorem 2. Consider the closed-loop transfer function (1), where $G_{0}$ is known. Assume $\left\{H\left(\omega_{k}\right)\right\}_{k=1}^{N}$ is known, (A1)-(A5) are satisfied, and for all $k \in \mathcal{N}, \sigma\left(\jmath \omega_{k}\right)=0$. For all $j \in \mathbb{Z}^{+}$, let $\Lambda_{j} \subseteq\left(\Psi \cap \mathcal{S}_{\rho}\right)$ be a finite set such that $\left\{\Lambda_{j}\right\}_{j=1}^{\infty}$ converges to $\Psi \cap \mathcal{S}_{\rho}$. For each $j \in \mathbb{Z}^{+}$, let $\beta_{j}^{+}$and $\phi_{j}^{+}$denote the identified parameters obtained from Algorithm 1 with the candidate pool $\Phi=\Lambda_{j}$. Then, for all $\epsilon>0$, there exists $L \in \mathbb{Z}^{+}$such that if $j>L$, then $\beta_{j}^{+} \in \mathbb{B}_{\epsilon}\left(\beta_{*}\right)$ and $\phi_{j}^{+} \in \mathbb{B}_{\epsilon}\left(\phi_{*}\right)$.

Proof. Let $\epsilon>0$. Since $\theta$ is continuous on $\mathcal{S}$ and $\phi_{*} \in \mathcal{S}$, it follows that there exists $\delta>0$ such that for all $x \in \mathbb{B}_{\delta}\left(\phi_{*}\right), \theta(x) \in \mathbb{B}_{\epsilon}\left(\theta\left(\phi_{*}\right)\right)$.

Define $\epsilon_{1} \triangleq \min \{\epsilon, \delta\}, \Lambda_{\mathrm{c}} \triangleq \overline{\Psi \cap \mathcal{S}_{\rho}}$, and

$$
\Lambda_{\epsilon_{1}} \triangleq \Lambda_{\mathrm{c}} \backslash \mathbb{B}_{\epsilon_{1}}\left(\phi_{*}\right)=\Lambda_{\mathrm{c}} \cap\left\{x \in \mathbb{R}^{d}:\left\|x-\phi_{*}\right\| \geq \epsilon_{1}\right\} .
$$

It follows from Proposition 8 that $\Lambda_{\mathrm{c}} \subseteq \mathcal{S}$ is compact. Since $\Lambda_{\mathrm{c}}$ is compact, and $\{x \in$ $\left.\mathbb{R}^{d}:\left\|x-\phi_{*}\right\| \geq \epsilon_{1}\right\}$ is closed, it follows from (16) that $\Lambda_{\epsilon_{1}}$ is compact.

Proposition 6 implies that $Q$ is continuous on $\Lambda_{\epsilon_{1}} \subseteq \Lambda_{\mathrm{c}} \subseteq \mathcal{S}$. Next, define $Q_{\epsilon_{1}} \triangleq$ $\min _{x \in \Lambda_{\epsilon_{1}}} Q(x)$, which exists because $Q$ is continuous on the compact set $\Lambda_{\epsilon_{1}}[33$, Theorem 7.7]. Assume for contradiction that $Q_{\epsilon_{1}}=0$. Thus, there exists $\phi \in \Lambda_{\epsilon_{1}}$ such that $Q(\phi)=0$. Since for all $k \in \mathcal{N}, H\left(\omega_{k}\right)=\tilde{G}\left(\jmath \omega_{k}\right)$, it follows from (8) and (15) that $0=Q(\phi)=J(\theta(\phi), \phi)=$ $\sum_{k=1}^{N}\left|\tilde{\mathcal{G}}\left(\jmath \omega_{k}, \theta(\phi), \phi\right)-\tilde{G}\left(\jmath \omega_{k}\right)\right|^{2}$. Thus, Proposition 5 implies that $\tilde{\mathcal{G}}(s, \theta(\phi), \phi) \equiv \tilde{G}(s)$, and it follows from (A5) that $\phi=\phi_{*} \notin \Lambda_{\epsilon_{1}}$, which is a contradiction. Thus, $Q_{\epsilon_{1}}>0$.

Next, since for all $k \in \mathcal{N}, \sigma\left(\jmath \omega_{k}\right)=0$, it follows from Proposition 4, (14), and (15) that $\beta_{*}=\theta\left(\phi_{*}\right)$ and $Q\left(\phi_{*}\right)=J\left(\beta_{*}, \phi_{*}\right)=0$. Furthermore, since $Q$ is continuous on $\Lambda_{\mathrm{c}}$, it follows that there exists $\delta_{1}>0$ such that for all $x \in \Lambda_{\mathrm{c}} \cap \mathbb{B}_{\delta_{1}}\left(\phi_{*}\right), Q(x)<Q_{\epsilon_{1}}$. Since $\left\{\Lambda_{j}\right\}_{j=1}^{\infty}$ converges to $\left(\Psi \cap \mathcal{S}_{\rho}\right) \subseteq \Lambda_{\mathrm{c}}$, it follows from Definition 1 that there exists a sequence $\left\{\phi_{j}: \phi_{j} \in \Lambda_{j}\right\}_{j=1}^{\infty}$ and $L \in \mathbb{Z}^{+}$such that for all $j>L, \phi_{j} \in \mathbb{B}_{\min \left\{\epsilon_{1}, \delta_{1}\right\}}\left(\phi_{*}\right) \subseteq \mathbb{B}_{\delta_{1}}\left(\phi_{*}\right)$. Thus, for all $j>L, Q\left(\phi_{j}\right)<Q_{\epsilon_{1}}$.

Let $j \in \mathbb{Z}^{+}$be such that $j>L$. It follows from Algorithm 1, (14), and (15) that $Q\left(\phi_{j}^{+}\right) \leq Q\left(\phi_{j}\right)<Q_{\epsilon_{1}}$. Assume for contradiction that $\phi_{j}^{+} \notin \mathbb{B}_{\epsilon_{1}}\left(\phi_{*}\right)$. Therefore, $\phi_{j}^{+} \in \Lambda_{\epsilon_{1}}$, which implies that $Q_{\epsilon_{1}} \leq Q\left(\phi_{j}^{+}\right)$, which is a contradiction. Thus, $\phi_{j}^{+} \in \mathbb{B}_{\epsilon_{1}}\left(\phi_{*}\right) \subseteq \mathbb{B}_{\epsilon}\left(\phi_{*}\right)$. Since $\phi_{j}^{+} \in \mathbb{B}_{\epsilon_{1}}\left(\phi_{*}\right) \subseteq \mathbb{B}_{\delta}\left(\phi_{*}\right)$, it follows that $\beta_{j}^{+}=\theta\left(\phi_{j}^{+}\right) \in \mathbb{B}_{\epsilon}\left(\theta\left(\phi_{*}\right)\right)=\mathbb{B}_{\epsilon}\left(\beta_{*}\right)$.

\section{Analysis with Noisy Frequency Response Data}

In this section, we analyze Algorithm 1 under the assumption of noisy frequency response data. Define $\hat{\Omega}_{0}: \mathcal{S} \times \mathbb{C}^{N} \rightarrow \mathbb{R}$ and $\hat{\Omega}_{1}: \mathcal{S} \times \mathbb{C}^{N} \rightarrow \mathbb{R}^{n_{1}+1}$ by

$$
\begin{aligned}
& \hat{\Omega}_{0}(\phi, \eta) \triangleq \sum_{k=1}^{N}\left|\frac{\tilde{\mathcal{N}}_{2}\left(\jmath \omega_{k}, \phi\right)}{\tilde{\mathcal{D}}\left(\jmath \omega_{k}, \phi\right)}-\tilde{G}\left(\jmath \omega_{k}\right)-\eta_{k}\right|^{2}, \\
& \hat{\Omega}_{1}(\phi, \eta) \triangleq 2 \operatorname{Re} \sum_{k=1}^{N}\left[\frac{\tilde{\mathcal{N}}_{1}\left(\jmath \omega_{k}, \phi\right)}{\tilde{\mathcal{D}}\left(\jmath \omega_{k}, \phi\right)}\right]^{*}\left[\frac{\tilde{\mathcal{N}}_{2}\left(\jmath \omega_{k}, \phi\right)}{\tilde{\mathcal{D}}\left(\jmath \omega_{k}, \phi\right)}-\tilde{G}\left(\jmath \omega_{k}\right)-\eta_{k}\right],
\end{aligned}
$$

where $\eta_{1}, \ldots, \eta_{N} \in \mathbb{C}$ and $\eta \triangleq\left[\begin{array}{lll}\eta_{1} & \ldots & \eta_{N}\end{array}\right]^{\mathrm{T}}$. Define $\sigma_{*} \triangleq\left[\begin{array}{llll}\sigma\left(\jmath \omega_{1}\right) & \cdots & \sigma\left(\jmath \omega_{N}\right)\end{array}\right]^{\mathrm{T}} \in$ $\mathbb{C}^{N}$, and note that $\hat{\Omega}_{0}\left(\phi, \sigma_{*}\right)=\Omega_{0}(\phi)$ and $\hat{\Omega}_{1}\left(\phi, \sigma_{*}\right)=\Omega_{1}(\phi)$. Thus, $\hat{\Omega}_{0}$ and $\hat{\Omega}_{1}$ are extensions of $\Omega_{0}$ and $\Omega_{1}$. Specifically, $\hat{\Omega}_{0}$ and $\hat{\Omega}_{1}$ are functions not only of the parameter $\phi$ but also the noise $\eta$. 
Define $\hat{J}: \mathbb{R}^{n_{1}+1} \times \mathcal{S} \times \mathbb{C}^{N} \rightarrow[0, \infty), \hat{\theta}: \mathcal{S} \times \mathbb{C}^{N} \rightarrow \mathbb{R}^{n_{1}+1}$, and $\hat{Q}: \mathcal{S} \times \mathbb{C}^{N} \rightarrow[0, \infty)$ by

$$
\begin{aligned}
\hat{J}(\beta, \phi, \eta) & \triangleq \sum_{k=1}^{N}\left|\tilde{\mathcal{G}}\left(\jmath \omega_{k}, \beta, \phi\right)-\tilde{G}\left(\jmath \omega_{k}\right)-\eta_{k}\right|^{2}, \\
\hat{\theta}(\phi, \eta) & \triangleq-\frac{1}{2} \Omega_{2}^{-1}(\phi) \hat{\Omega}_{1}(\phi, \eta), \\
\hat{Q}(\phi, \eta) & \triangleq \hat{J}(\hat{\theta}(\phi, \eta), \phi, \eta) .
\end{aligned}
$$

Note that $\hat{J}\left(\beta, \phi, \sigma_{*}\right)=J(\beta, \phi), \hat{\theta}\left(\phi, \sigma_{*}\right)=\theta(\phi)$, and $\hat{Q}\left(\phi, \sigma_{*}\right)=Q(\phi)$. Thus, $\hat{J}, \hat{\theta}$, and $\hat{Q}$ are extensions of $J, \theta$, and $Q$. Specifically, $\hat{J}, \hat{\theta}$, and $\hat{Q}$ are functions not only of the parameters $\beta$ and $\phi$ but also of the noise $\eta$.

It follows from (4), (11), and (17)-(19) that

$$
\hat{J}(\beta, \phi, \eta)=\beta^{\mathrm{T}} \Omega_{2}(\phi) \beta+\hat{\Omega}_{1}^{\mathrm{T}}(\phi, \eta) \beta+\hat{\Omega}_{0}(\phi, \eta) .
$$

Furthermore, it follows from (14), (15), (19)-(21), and Proposition 4 that

$$
\begin{aligned}
& \hat{\theta}\left(\phi_{*}, 0\right)=-\frac{1}{2} \Omega_{2}^{-1}\left(\phi_{*}\right) \hat{\Omega}_{1}\left(\phi_{*}, 0\right)=\beta_{*}, \\
& \hat{Q}\left(\phi_{*}, 0\right)=\hat{J}\left(\beta_{*}, \phi_{*}, 0\right)=0 .
\end{aligned}
$$

The following result is an extension of Proposition 6. The proof is in Appendix C.

Proposition 9. Assume (A1)-(A3) are satisfied. Then, $\hat{\theta}$ and $\hat{Q}$ are continuous on $\mathcal{S} \times \mathbb{C}^{N}$.

\section{1. $\phi_{*}$ in the candidate pool $\Phi$}

The following result provides sufficient conditions such that the identified parameter $\phi^{+}$ equals $\phi_{*}$. This result also shows that if the norm of the noise $\sigma_{*}$ is sufficiently small, then the identified parameter $\beta^{+}$is arbitrarily close to $\beta_{*}$.

Theorem 3. Consider the closed-loop transfer function (1), where $G_{0}$ is known. Assume $\left\{H\left(\omega_{k}\right)\right\}_{k=1}^{N}$ is known and (A1)-(A5) are satisfied. Furthermore, consider Algorithm 1 with the candidate pool $\Phi \subseteq(\Psi \cap \mathcal{S})$, and assume $\phi_{*} \in \Phi$. Let $\beta^{+}$and $\phi^{+}$denote the identified parameters obtained from Algorithm 1. Then, there exists $\delta_{0}>0$ such that if $\left\|\sigma_{*}\right\|<\delta_{0}$, then $\phi^{+}=\phi_{*}$. Furthermore, for all $\epsilon>0$, there exists $\delta \in\left(0, \delta_{0}\right)$ such that if $\left\|\sigma_{*}\right\|<\delta$, then $\beta^{+} \in \mathbb{B}_{\epsilon}\left(\beta_{*}\right)$.

Proof. Let $\phi \in \Phi \backslash\left\{\phi_{*}\right\}$, and assume for contradiction that $\hat{Q}(\phi, 0)=0$. It follows from (19) and (21) that $0=\hat{Q}(\phi, 0)=\hat{J}(\hat{\theta}(\phi, 0), \phi, 0)=\sum_{k=1}^{N}\left|\tilde{\mathcal{G}}\left(\jmath \omega_{k}, \hat{\theta}(\phi, 0), \phi\right)-\tilde{G}\left(\jmath \omega_{k}\right)\right|^{2}$. Thus, Proposition 5 implies that $\tilde{\mathcal{G}}(s, \hat{\theta}(\phi, 0), \phi) \equiv \tilde{G}(s)$, and it follows from (A5) that $\phi=\phi_{*}$, which is a contradiction. Therefore, $\hat{Q}(\phi, 0)>0$.

Define $U \triangleq \min _{x \in \Phi \backslash\left\{\phi_{*}\right\}} \hat{Q}(x, 0)>0$. Since $\hat{Q}$ is continuous on $\mathcal{S} \times \mathbb{C}^{N}$, it follows that for each $i \in \mathcal{M}, \hat{Q}\left(\phi_{i}, \cdot\right)$ is continuous on $\mathbb{C}^{N}$. Thus, for each $i \in \mathcal{M}$, there exists $\delta_{i}>0$ such that for all $\eta \in\left\{x \in \mathbb{C}^{N}:\|x\|<\delta_{i}\right\}$,

$$
\left|\hat{Q}\left(\phi_{i}, \eta\right)-\hat{Q}\left(\phi_{i}, 0\right)\right|<U / 2 \text {. }
$$

Define $\delta_{0} \triangleq \min _{i \in \mathcal{M}} \delta_{i}>0$, and assume that $\left\|\sigma_{*}\right\|<\delta_{0}$. Since $\phi_{*} \in \Phi$, it follows that there exists $m \in \mathcal{M}$ such that $\phi_{m}=\phi_{*}$. Since $\hat{Q}\left(\phi_{m}, 0\right)=\hat{Q}\left(\phi_{*}, 0\right)=0$, it follows from (25) that $\hat{Q}\left(\phi_{m}, \sigma_{*}\right)=\left|\hat{Q}\left(\phi_{m}, \sigma_{*}\right)-\hat{Q}\left(\phi_{m}, 0\right)\right|<U / 2$. Let $j \in \mathcal{M} \backslash\{m\}$. It follows from (25) that $-U / 2<\hat{Q}\left(\phi_{j}, \sigma_{*}\right)-\hat{Q}\left(\phi_{j}, 0\right)$, which implies that $\hat{Q}\left(\phi_{j}, \sigma_{*}\right)>\hat{Q}\left(\phi_{j}, 0\right)-U / 2$. Since, in addition, $\hat{Q}\left(\phi_{j}, 0\right) \geq U$, it follows that $\hat{Q}\left(\phi_{j}, \sigma_{*}\right)>U / 2$. Therefore, $\hat{Q}\left(\phi_{m}, \sigma_{*}\right)<\hat{Q}\left(\phi_{j}, \sigma_{*}\right)$, 
which implies that $Q\left(\phi_{m}\right)<Q\left(\phi_{j}\right)$. Thus, (13), (14), (15), and Proposition 3 imply that $\mathcal{J}_{m}\left(\beta_{m}\right)<\mathcal{J}_{j}\left(\beta_{j}\right)$. Therefore, it follows from Algorithm 1 that $\phi^{+}=\phi_{m}=\phi_{*}$ and

$$
\beta^{+}=\beta_{m}=\theta\left(\phi_{*}\right)=\hat{\theta}\left(\phi_{*}, \sigma_{*}\right) .
$$

Let $\epsilon>0$. Since $\hat{\theta}$ is continuous on $\mathcal{S} \times \mathbb{C}^{N}$, it follows that $\hat{\theta}\left(\phi_{*}, \cdot\right)$ is continuous on $\mathbb{C}^{N}$. Therefore, there exists $\delta \in\left(0, \delta_{0}\right)$ such that for all $\eta \in\left\{x \in \mathbb{C}^{N}:\|x\|<\delta\right\}, \hat{\theta}\left(\phi_{*}, \eta\right) \in$ $\mathbb{B}_{\epsilon}\left(\hat{\theta}\left(\phi_{*}, 0\right)\right)$. Finally, assume $\left\|\sigma_{*}\right\|<\delta$, and (23) and (26) imply that $\beta^{+} \in \mathbb{B}_{\epsilon}\left(\beta_{*}\right)$.

\section{2. $\phi_{*}$ not necessarily in the candidate pool $\Phi$}

We now extend the analysis in the previous section to address the case where $\phi_{*}$ is not necessarily in the candidate pool. In the following result, we consider Algorithm 1 with a sequence of candidate pools that converges to $\Psi \cap \mathcal{S}_{\rho}$. This result demonstrates that a sufficiently dense candidate pool and sufficiently small noise $\sigma_{*}$ yield identified parameters $\beta^{+}$and $\phi^{+}$that are arbitrarily close to $\beta_{*}$ and $\phi_{*}$.

Theorem 4. Consider the closed-loop transfer function (1), where $G_{0}$ is known. Assume $\left\{H\left(\omega_{k}\right)\right\}_{k=1}^{N}$ is known and (A1)-(A5) are satisfied. For all $j \in \mathbb{Z}^{+}$, let $\Lambda_{j} \subseteq\left(\Psi \cap \mathcal{S}_{\rho}\right)$ be a finite set such that $\left\{\Lambda_{j}\right\}_{j=1}^{\infty}$ converges to $\Psi \cap \mathcal{S}_{\rho}$. For each $j \in \mathbb{Z}^{+}$, let $\beta_{j}^{+}$and $\phi_{j}^{+}$denote the identified parameters obtained from Algorithm 1 with the candidate pool $\Phi=\Lambda_{j}$. Then, for all $\epsilon>0$, there exist $\delta>0$ and $L \in \mathbb{Z}^{+}$such that if $\left\|\sigma_{*}\right\|<\delta$ and $j>L$, then $\beta_{j}^{+} \in \mathbb{B}_{\epsilon}\left(\beta_{*}\right)$ and $\phi_{j}^{+} \in \mathbb{B}_{\epsilon}\left(\phi_{*}\right)$.

Proof. Let $\epsilon>0$. Since $\hat{\theta}$ is continuous on $\mathcal{S} \times \mathbb{C}^{N}$ and $\phi_{*} \in \mathcal{S}$, it follows that there exists $\delta_{0}>0$ such that for all $\phi \in \mathbb{B}_{\delta_{0}}\left(\phi_{*}\right)$ and all $\eta \in\left\{x \in \mathbb{C}^{N}:\|x\|<\delta_{0}\right\}$,

$$
\hat{\theta}(\phi, \eta) \in \mathbb{B}_{\epsilon}\left(\hat{\theta}\left(\phi_{*}, 0\right)\right) \text {. }
$$

Define $\epsilon_{1} \triangleq \min \left\{\epsilon, \delta_{0}\right\}, \Lambda_{\mathrm{c}} \triangleq \overline{\Psi \cap \mathcal{S}_{\rho}}$, and

$$
\Lambda_{\epsilon_{1}} \triangleq \Lambda_{\mathrm{c}} \backslash \mathbb{B}_{\epsilon_{1}}\left(\phi_{*}\right)=\Lambda_{\mathrm{c}} \cap\left\{x \in \mathbb{R}^{d}:\left\|x-\phi_{*}\right\| \geq \epsilon_{1}\right\} .
$$

It follows from Proposition 8 that $\Lambda_{\mathrm{c}} \subseteq \mathcal{S}$ is compact. Since $\Lambda_{\mathrm{c}}$ is compact, and $\{x \in$ $\left.\mathbb{R}^{d}:\left\|x-\phi_{*}\right\| \geq \epsilon_{1}\right\}$ is closed, it follows from (28) that $\Lambda_{\epsilon_{1}}$ is compact.

Let $v>\delta_{0}$, and define $V \triangleq\left\{x \in \mathbb{C}^{N}:\|x\| \leq v\right\}$. Since $\Lambda_{\epsilon_{1}} \subseteq \Lambda_{\mathrm{c}} \subseteq \mathcal{S}$ and $V \subseteq \mathbb{C}^{N}$, it follows from Proposition 9 that $\hat{Q}$ is continuous on $\Lambda_{\epsilon_{1}} \times V$. Next, define $\Theta: V \rightarrow[0, \infty)$ by $\Theta(\eta) \triangleq \min _{\phi \in \Lambda_{\epsilon_{1}}} \hat{Q}(\phi, \eta)$, which exist because $\Lambda_{\epsilon_{1}}$ is compact and $\hat{Q}$ is continuous on $\Lambda_{\epsilon_{1}} \times V$ [33, Theorem 7.7].

Assume for contradiction that $\Theta(0)=0$. Thus, there exists $z \in \Lambda_{\epsilon_{1}}$ such that $\hat{Q}(z, 0)=0$, and it follows from (19) and (21) that $0=\hat{J}(\hat{\theta}(z, 0), z, 0)=\sum_{k=1}^{N}\left|\tilde{\mathcal{G}}\left(\jmath \omega_{k}, \hat{\theta}(z, 0), z\right)-\tilde{G}\left(\jmath \omega_{k}\right)\right|^{2}$. Thus, Proposition 5 implies that $\tilde{\mathcal{G}}(s, \hat{\theta}(z, 0), z) \equiv \tilde{G}(s)$, and it follows from (A5) that $z=$ $\phi_{*} \notin \Lambda_{\epsilon_{1}}$, which is a contradiction. Thus, $\Theta(0)>0$.

Since $\hat{Q}$ is continuous on $\Lambda_{\epsilon_{1}} \times V$, and $\Lambda_{\epsilon_{1}}$ and $V$ are compact, it follows from [34, Theorem 9.14] that $\Theta$ is continuous on $V$. Furthermore, since $\hat{Q}$ is continuous on $\mathcal{S} \times \mathbb{C}^{N}$, it follows that $\hat{Q}\left(\phi_{*}, \cdot\right)$ is continuous on $V$. Thus, $W: V \rightarrow \mathbb{R}$ defined by $W(\eta) \triangleq \Theta(\eta)-\hat{Q}\left(\phi_{*}, \eta\right)$ is continuous on $V$. It follows from $(24)$ that $W(0)=\Theta(0)-\hat{Q}\left(\phi_{*}, 0\right)=\Theta(0)>0$. Since, in addition, $W$ is continuous on $V$, it follows that there exists $\delta_{1} \in(0, v)$ such that for all $\eta \in\left\{x \in \mathbb{C}^{N}:\|x\|<\delta_{1}\right\}, W(\eta)>0$. Define $\delta \triangleq \min \left\{\delta_{0}, \delta_{1}\right\}>0$ and assume $\left\|\sigma_{*}\right\|<\delta$.

Since $W\left(\sigma_{*}\right)>0$ and Proposition 9 implies that $\hat{Q}\left(\cdot, \sigma_{*}\right)$ is continuous on $\Lambda_{\mathrm{c}}$, it follows from the continuity of $\hat{Q}\left(\cdot, \sigma_{*}\right)$ that there exists $\delta_{2}>0$ such that for all $\phi \in\left(\Lambda_{\mathrm{c}} \cap \mathbb{B}_{\delta_{2}}\left(\phi_{*}\right)\right)$, $\left|\hat{Q}\left(\phi, \sigma_{*}\right)-\hat{Q}\left(\phi_{*}, \sigma_{*}\right)\right|<W\left(\sigma_{*}\right)$. Thus, for all $\phi \in\left(\Lambda_{\mathrm{c}} \cap \mathbb{B}_{\delta_{2}}\left(\phi_{*}\right)\right), \hat{Q}\left(\phi, \sigma_{*}\right)-\hat{Q}\left(\phi_{*}, \sigma_{*}\right) \leq$ $\left|\hat{Q}\left(\phi, \sigma_{*}\right)-\hat{Q}\left(\phi_{*}, \sigma_{*}\right)\right|<W\left(\sigma_{*}\right)=\Theta\left(\sigma_{*}\right)-\hat{Q}\left(\phi_{*}, \sigma_{*}\right)$, which implies that

$$
\hat{Q}\left(\phi, \sigma_{*}\right)<\Theta\left(\sigma_{*}\right) \text {. }
$$


Since $\left\{\Lambda_{j}\right\}_{j=1}^{\infty}$ converges to $\left(\Psi \cap \mathcal{S}_{\rho}\right) \subseteq \Lambda_{\mathrm{c}}$, it follows from Definition 1 that there exists a sequence $\left\{\phi_{j}: \phi_{j} \in \Lambda_{j}\right\}_{j=1}^{\infty}$ and $L \in \mathbb{Z}^{+}$such that for all $j>L, \phi_{j} \in \mathbb{B}_{\min \left\{\epsilon_{1}, \delta_{2}\right\}}\left(\phi_{*}\right)$. Thus, it follows from (29) that for all $j>L, \hat{Q}\left(\phi_{j}, \sigma_{*}\right)<\Theta\left(\sigma_{*}\right)$.

Let $j \in \mathbb{Z}^{+}$be such that $j>L$. It follows from Algorithm 1, (14), and (15) that $Q\left(\phi_{j}^{+}\right) \leq Q\left(\phi_{j}\right)$. Therefore, (29) implies that $\hat{Q}\left(\phi_{j}^{+}, \sigma_{*}\right) \leq \hat{Q}\left(\phi_{j}, \sigma_{*}\right)<\Theta\left(\sigma_{*}\right)$. Assume for contradiction that $\phi_{j}^{+} \notin \mathbb{B}_{\epsilon_{1}}\left(\phi_{*}\right)$. Therefore, $\phi_{j}^{+} \in \Lambda_{\epsilon_{1}}$, which implies that $\Theta\left(\sigma_{*}\right)=$ $\min _{\phi \in \Lambda_{\epsilon_{1}}} \hat{Q}\left(\phi, \sigma_{*}\right) \leq \hat{Q}\left(\phi_{j}^{+}, \sigma_{*}\right)$, which is a contradiction. Thus, $\phi_{j}^{+} \in \mathbb{B}_{\epsilon_{1}}\left(\phi_{*}\right) \subseteq \mathbb{B}_{\epsilon}\left(\phi_{*}\right)$. Since $\phi_{j}^{+} \in \mathbb{B}_{\epsilon_{1}}\left(\phi_{*}\right) \subseteq \mathbb{B}_{\delta_{0}}\left(\phi_{*}\right)$, it follows from (23) and (27) that $\beta_{j}^{+}=\hat{\theta}\left(\phi_{j}^{+}, \sigma_{*}\right) \in \mathbb{B}_{\epsilon}\left(\hat{\theta}\left(\phi_{*}, 0\right)\right)=$ $\mathbb{B}_{\epsilon}\left(\beta_{*}\right)$.

\section{Numerical Examples}

For all examples in this section, let $G_{0}(s)=4 /(s+2), G_{1}(s)=(2.1 s+3) /(s+6.5)$, and $G_{2}(s)=5.4 /(s+7.1)$, which implies that $\beta_{*}=\left[\begin{array}{ll}2.1 & 3\end{array}\right]^{\mathrm{T}}$ and $\phi_{*}=\left[\begin{array}{lll}6.5 & 5.4 & 7.1\end{array}\right]^{\mathrm{T}}$. Let $\Psi=[-8,8] \times[-8,8] \times[-8,8]$, which is a compact and perfect set containing $\phi_{*}$. We now show that this example satisfies (A5). Let $\beta=\left[\begin{array}{ll}u_{1} & u_{2}\end{array}\right]^{\mathrm{T}} \in \mathbb{R}^{2}$ and $\phi=\left[\begin{array}{lll}v_{1} & v_{2} & v_{3}\end{array}\right]^{\mathrm{T}} \in \mathbb{R}^{3}$, and assume $\tilde{\mathcal{G}}(s, \beta, \phi) \equiv \tilde{G}(s)$, where $\tilde{G}$ is given by (1). It follows that

$$
\tilde{\mathcal{G}}(s, \beta, \phi)=\frac{4\left[\left(u_{1} s+u_{2}\right)\left(s+v_{3}\right)+v_{2}\left(s+v_{1}\right)\right]}{\left(s+v_{1}\right)\left[(s+2)\left(s+v_{3}\right)+4 v_{2}\right]}=\frac{4\left(2.1 s^{2}+23.31 s+56.4\right)}{(s+6.5)\left(s^{2}+9.1 s+35.8\right)} .
$$

Since the roots of $s^{2}+9.1 s+35.8$ are complex, (30) implies that $v_{1}=6.5$ and $(s+2)(s+$ $\left.v_{3}\right)+4 v_{2}=s^{2}+9.1 s+35.8$, or equivalently, $v_{3}=7.1$ and $v_{2}=5.4$. Moreover, the numerator of (30) implies that $\left(u_{1} s+u_{2}\right)\left(s+v_{3}\right)+v_{2}\left(s+v_{1}\right)=2.1 s^{2}+23.31 s+56.4$, or equivalently, $u_{1}=2.1$ and $u_{2}=3$. Thus, $\beta=\beta_{*}$ and $\phi=\phi_{*}$.

For all examples in this section, let $N=20$ and $\omega_{k}=0.2 \pi k$, where $k \in \mathcal{N}$.

Example 2. Noiseless data and $\phi_{*} \in \Phi$. Assume $\sigma\left(\jmath \omega_{k}\right) \equiv 0$. Consider $\Pi_{0} \triangleq\left\{\phi \in \mathbb{R}^{3}\right.$ : $\left.E_{1} \phi, E_{2} \phi, E_{3} \phi \in\{-8+0.1 k\}_{k=0}^{160}\right\} \subseteq \Psi$, define $\Lambda_{0} \triangleq \Pi_{0} \cap \mathcal{S}$, and note that $\phi_{*} \in \Lambda_{0}$. Algorithm 1 is used with the candidate pool $\Phi=\Lambda_{0}$ to obtain $\beta^{+}$and $\phi^{+}$. The identified parameters are $\beta^{+}=\beta_{*}=\left[\begin{array}{ll}2.1 & 3\end{array}\right]^{\mathrm{T}}$ and $\phi^{+}=\phi_{*}=\left[\begin{array}{lll}6.5 & 5.4 & 7.1\end{array}\right]^{\mathrm{T}}$, which agrees with Theorem 1.

Example 3. Noiseless data and $\phi_{*} \notin \Phi$. Assume $\sigma\left(\jmath \omega_{k}\right) \equiv 0$. For $j=1, \ldots, 25$, consider

$$
\Pi_{j} \triangleq\left\{\phi \in \mathbb{R}^{3}: E_{1} \phi, E_{2} \phi, E_{3} \phi \in\left\{-8+\frac{16}{5+10(j-1)} k\right\}_{k=0}^{5+10(j-1)}\right\} \subseteq \Psi,
$$

define $\Lambda_{j} \triangleq \Pi_{j} \cap \mathcal{S}_{\rho}$, where $\rho=-0.001$, and note that for $j=1, \ldots, 25, \phi_{*} \notin \Lambda_{j}$.

For $j=1, \ldots, 25$, Algorithm 1 is used with the candidate pool $\Phi=\Lambda_{j}$ to obtain the identified parameters $\beta_{j}^{+}$and $\phi_{j}^{+}$. Figure 3 demonstrates that $\left\|\beta_{j}^{+}-\beta_{*}\right\|_{2}$ and $\left\|\phi_{j}^{+}-\phi_{*}\right\|_{2}$ are arbitrarily small for sufficient large $j$, which agrees with Theorem 2 . Note that $\left\|\beta_{j}^{+}-\beta_{*}\right\|_{2}$ and $\left\|\phi_{j}^{+}-\phi_{*}\right\|_{2}$ do not decrease monotonically. The Bode plots of $G_{1}$ and $G_{2}$, and the identified transfer functions $G_{1}^{+}$and $G_{2}^{+}$with $\Phi=\Lambda_{1}, \Phi=\Lambda_{2}$, and $\Phi=\Lambda_{25}$, are shown in Figure 4. The identified transfer functions $G_{1}^{+}$and $G_{2}^{+}$with $\Phi=\Lambda_{25}$ approximate $G_{1}$ and $G_{2}$ better than those with $\Phi=\Lambda_{1}$ and $\Phi=\Lambda_{2}$.

Example 4. Noisy data and $\phi_{*} \in \Phi$. For $i=1, \ldots, 20$, let $n_{r, i}(t)$ and $n_{y, i}(t)$ be zeromean Gaussian white-noise realizations with variance of $4^{-i}$. Moreover, for $i=1, \ldots, 20$, let $\sigma_{r, i}(\jmath \omega)$ and $\sigma_{y, i}(\jmath \omega)$ be the Fourier transforms of $n_{r, i}$ and $n_{y, i}$, respectively. For $i=$ $1, \ldots, 20$, the noisy closed-loop frequency response data is $H_{i}\left(\omega_{k}\right) \triangleq \tilde{G}\left(\jmath \omega_{k}\right)+\sigma_{i}\left(\jmath \omega_{k}\right)$, where 

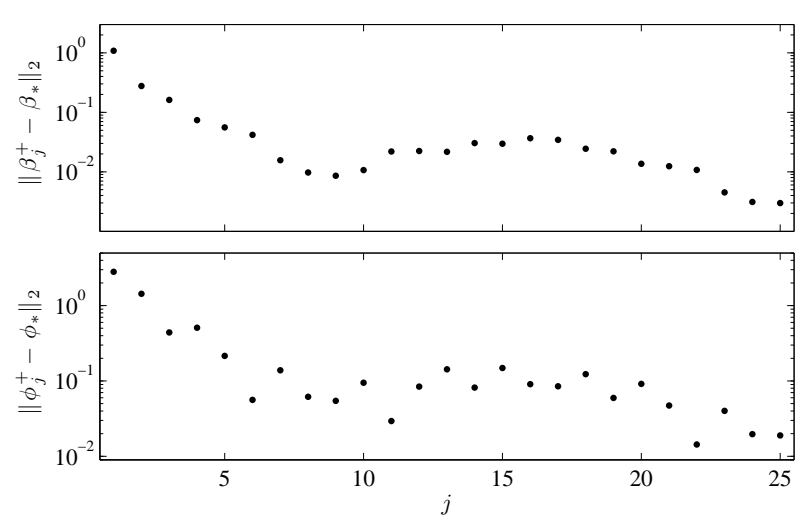

Figure 3: Noiseless data and $\phi_{*} \notin \Phi$. For $j=$ $1, \ldots, 25$, Algorithm 1 is used with the candidate pool $\Lambda_{j}$ to obtain the identified parameters $\beta_{j}^{+}$and $\phi_{j}^{+}$. Note that $\left\|\beta_{j}^{+}-\beta_{*}\right\|_{2}$ and $\left\|\phi_{j}^{+}-\phi_{*}\right\|_{2}$ can be made arbitrarily small if the candidate pool is sufficiently dense.
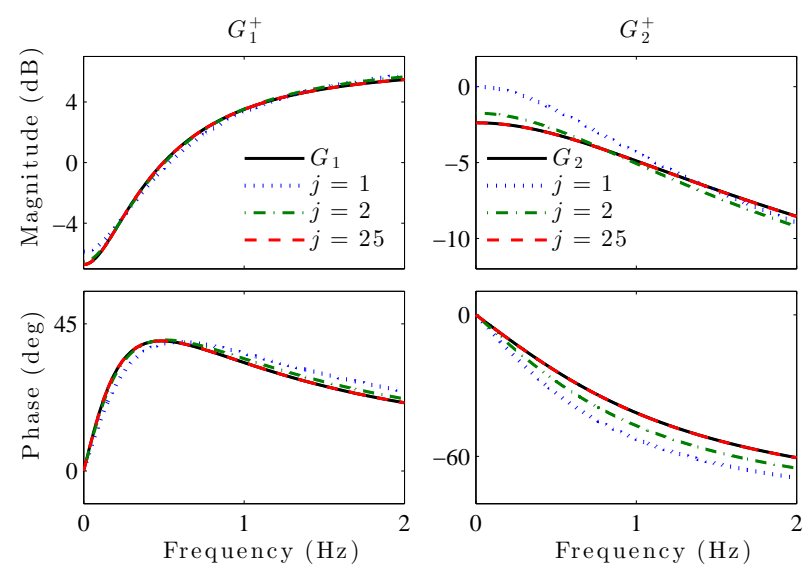

Figure 4: Noiseless data and $\phi_{*} \notin \Phi$. For $j=1,2,25$, Algorithm 1 is used with the candidate pool $\Lambda_{j}$ to obtain the identified transfer functions $G_{1}^{+}$and $G_{2}^{+}$. Note that $G_{1}^{+}$and $G_{2}^{+}$with candidate pool $\Lambda_{25}$ approximate $G_{1}$ and $G_{2}$ better than those with candidate pools $\Lambda_{1}$ and $\Lambda_{2}$.

$\sigma_{i}\left(\jmath \omega_{k}\right) \triangleq\left[\tilde{G}\left(\jmath \omega_{k}\right) \sigma_{r, i}\left(\jmath \omega_{k}\right)+\sigma_{y, i}\left(\jmath \omega_{k}\right)\right] / r\left(\jmath \omega_{k}\right)$. For $i=1, \ldots, 20$, define $R_{i} \triangleq \frac{1}{N} \sum_{k=1}^{N}\left|\frac{\sigma_{i}\left(\jmath \omega_{k}\right)}{\tilde{G}\left(\jmath \omega_{k}\right)}\right|$, which is the frequency-averaged noise-to-signal ratio. In this example, for $i=1, \ldots, 20$, $R_{i} \in(0,24)$. Specifically, $R_{1}=23.8, R_{5}=1.39, R_{10}=4.49 \times 10^{-2}, R_{12}=9.53 \times 10^{-3}$, and $R_{20}=4.36 \times 10^{-5}$.

For $i=1, \ldots, 20$, Algorithm 1 is used with the candidate pool $\Phi=\Lambda_{0}$ and data $\left\{H_{i}\left(\omega_{k}\right)\right\}_{k=1}^{N}$ to obtain the identified parameters $\beta_{i}^{+}$and $\phi_{i}^{+}$. Figure 5 demonstrates that for $i \geq 14, \phi_{i}^{+}=\phi_{*}$ and for sufficiently large $i,\left\|\beta_{i}^{+}-\beta_{*}\right\|_{2}$ is arbitrarily small, which agrees with Theorem 3. The Bode plots of $G_{1}$ and $G_{2}$, and $G_{1}^{+}$and $G_{2}^{+}$with data $\left\{H_{5}\left(\omega_{k}\right)\right\}_{k=1}^{N}$, $\left\{H_{10}\left(\omega_{k}\right)\right\}_{k=1}^{N}$, and $\left\{H_{20}\left(\omega_{k}\right)\right\}_{k=1}^{N}$ are shown in Figure 6 . The identified transfer functions $G_{1}^{+}$and $G_{2}^{+}$with data $\left\{H_{20}\left(\omega_{k}\right)\right\}_{k=1}^{N}$ approximate $G_{1}$ and $G_{2}$ better than those with data $\left\{H_{5}\left(\omega_{k}\right)\right\}_{k=1}^{N}$ and $\left\{H_{10}\left(\omega_{k}\right)\right\}_{k=1}^{N}$. The identified transfer function $G_{2}^{+}$with data $\left\{H_{20}\left(\omega_{k}\right)\right\}_{k=1}^{N}$ is $G_{2}^{+}=G_{2}$.
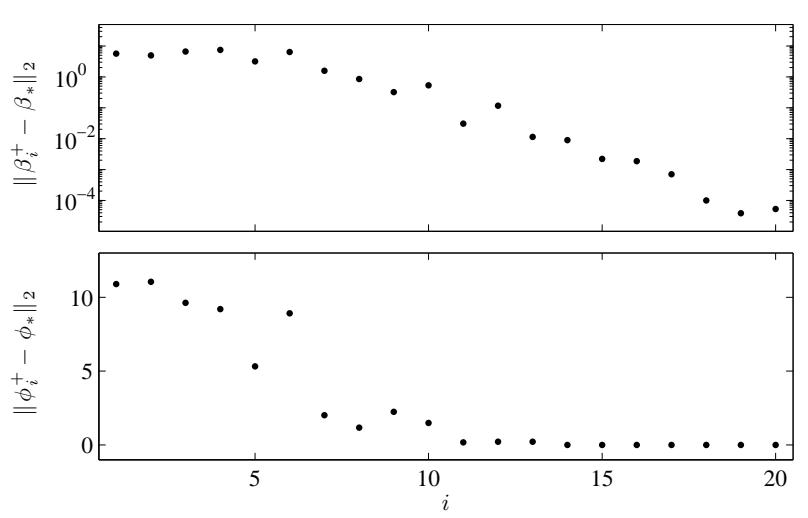

Figure 5: Noisy data and $\phi_{*} \in \Phi$. For $i=1, \ldots, 20$, Algorithm 1 is used with the candidate pool $\Lambda_{0}$ and data $\left\{H_{i}\left(\omega_{k}\right)\right\}_{k=1}^{N}$ to obtain the identified parameters $\beta_{i}^{+}$and $\phi_{i}^{+}$. For $i \geq 14, \phi_{i}^{+}=\phi_{*}$. Note that $\| \beta_{i}^{+}-$ $\beta_{*} \|_{2}$ can be made arbitrarily small if the norm of the noise $\sigma_{*}$ is sufficiently small.
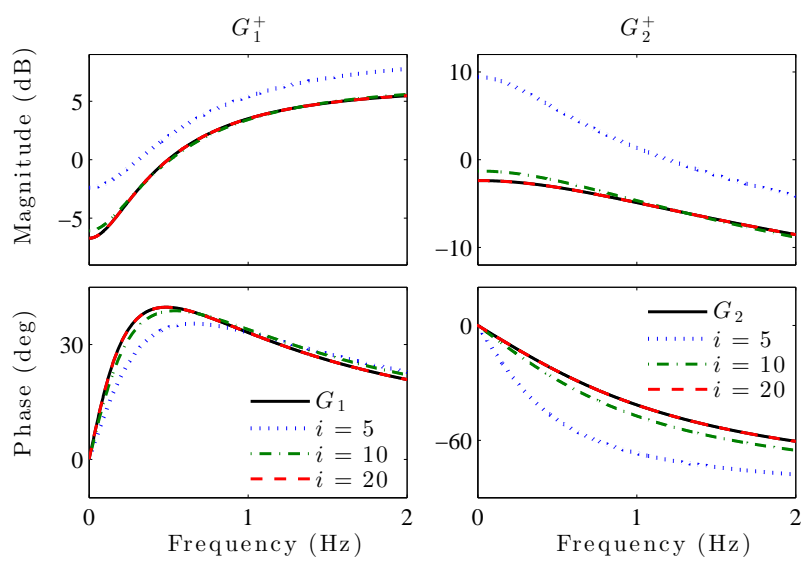

Figure 6: Noisy data and $\phi_{*} \in \Phi$. For $i=5,10,20$, Algorithm 1 is used with the candidate pool $\Lambda_{0}$ and data $\left\{H_{i}\left(\omega_{k}\right)\right\}_{k=1}^{N}$ to obtain the identified transfer functions $G_{1}^{+}$and $G_{2}^{+}$. Note that $G_{1}^{+}$and $G_{2}^{+}$with $\left\{H_{20}\left(\omega_{k}\right)\right\}_{k=1}^{N}$ approximate $G_{1}$ and $G_{2}$ better than those with $\left\{H_{5}\left(\omega_{k}\right)\right\}_{k=1}^{N}$ and $\left\{H_{10}\left(\omega_{k}\right)\right\}_{k=1}^{N}$. 
Example 5. Noisy data and $\phi_{*} \notin \Phi$. Consider the noisy closed-loop frequency response data $\left\{H_{5}\left(\omega_{k}\right)\right\}_{k=1}^{N},\left\{H_{10}\left(\omega_{k}\right)\right\}_{k=1}^{N},\left\{H_{12}\left(\omega_{k}\right)\right\}_{k=1}^{N}$, and $\left\{H_{20}\left(\omega_{k}\right)\right\}_{k=1}^{N}$ given in Example 4. Moreover, consider $\Lambda_{1}, \ldots, \Lambda_{25}$ given in Example 3. For $i=5,10,12,20$, and $j=1, \ldots, 25$, Algorithm 1 is used with the candidate pool $\Phi=\Lambda_{j}$ and data $\left\{H_{i}\left(\omega_{k}\right)\right\}_{k=1}^{N}$ to obtain the identified parameters $\beta_{j, i}^{+}$and $\phi_{j, i}^{+}$. Figure 7 demonstrates that $\left\|\beta_{j, i}^{+}-\beta_{*}\right\|_{2}$ and $\left\|\phi_{j, i}^{+}-\phi_{*}\right\|_{2}$ are arbitrarily small for sufficient large $j$ and $i$, which agrees with Theorem 4 .
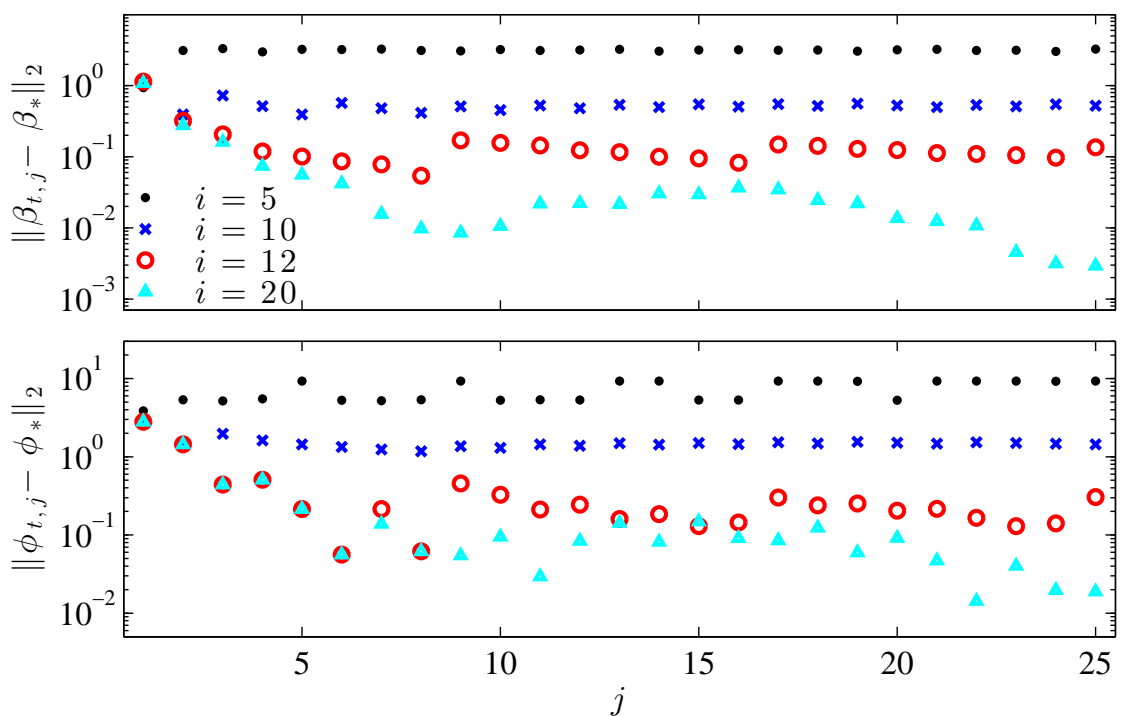

Figure 7: Noisy data and $\phi_{*} \notin \Phi$. For $i=5,10,12,20$, and $j=1, \ldots, 25$, Algorithm 1 is used with the candidate pool $\Lambda_{j}$ and data $\left\{H_{i}\left(\omega_{k}\right)\right\}_{k=1}^{N}$ to obtain the identified parameters $\beta_{j, i}^{+}$and $\phi_{j, i}^{+}$. Note that $\left\|\beta_{j, i}^{+}-\beta_{*}\right\|_{2}$ and $\left\|\phi_{j, i}^{+}-\phi_{*}\right\|_{2}$ are small for large $j$ and $i$.

\section{Application to Modeling Human Control Behavior}

In this section, we apply Algorithm 1 to data from an experiment in which human subjects learn to interact with a dynamic system. To demonstrate the SSID method, we use data for a single subject and model that subject's feedback and feedforward control behavior. In the experiment, a total of ten subjects, who are 18-35 years of age and have no known neurological or motor-control disorders, interact with a dynamic system 40 times over a 2-week period. During each interaction, a subject uses a single-degree-of-freedom joystick to control the motion of an object that is displayed on a computer screen. The object's position $y_{\mathrm{t}}$ is the output of a dynamic system $G_{0}$, and the joystick position $u_{\mathrm{t}}$ is the input to the dynamic system $G_{0}$. The subject has no initial knowledge of the dynamics $G_{0}$ between $u_{\mathrm{t}}$ and $y_{\mathrm{t}}$. A reference object, whose position $r_{\mathrm{t}}$ is independent of $u_{\mathrm{t}}$, is also displayed on the computer screen. The feedback $y_{\mathrm{t}}$ and feedforward $r_{\mathrm{t}}$ are provided visually on the computer screen, using sliding needle displays as shown in Figure 8. The subject's objective is to move the joystick and thus generate a control $u_{\mathrm{t}}$ that minimizes the magnitude of the command-following error $e_{\mathrm{t}}=r_{\mathrm{t}}-y_{\mathrm{t}}$.

This experiment satisfies the U.S. Department of Health and Human Services Code of Federal Regulation for human subject research (45 CFR 46) and was approved by the University of Kentucky Institutional Review Board (IRB number 12-0816-P4S). The experimental methods are related to those of reaching experiments (e.g., $[6,7,10]$ ), which are used extensively to study human motor learning. We also note that computer-based experiments with visual feedback have been used to study motor learning [20, 30, 31]. For example, in [20], a subject also uses a joystick to control the position of an object displayed on a computer 
screen. However, [20] examines feedback behavior only and is restricted to plants that are either static or have pure integrator dynamics.

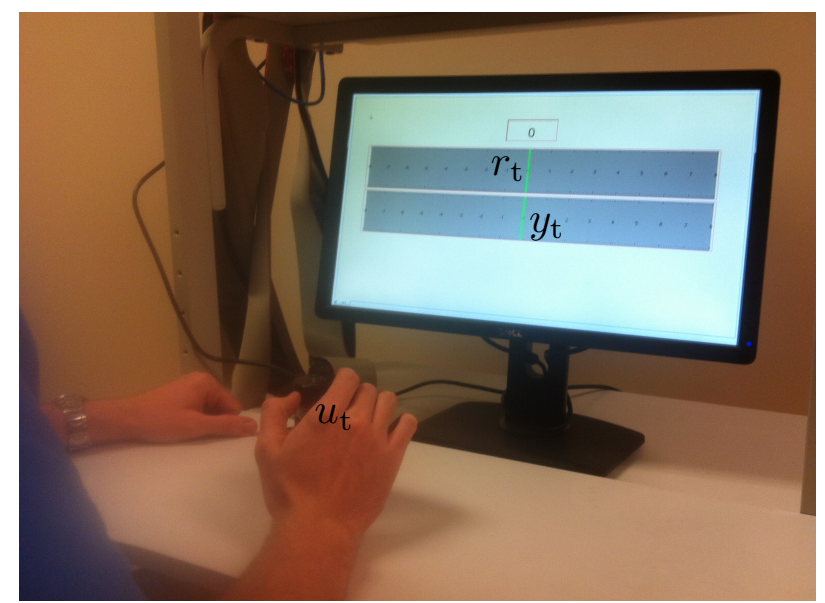

Figure 8: Subjects receive visual feedback $y_{\mathrm{t}}$ and feedforward $r_{\mathrm{t}}$.

In this experiment, the plant $G_{0}$ is the two-degree-of-freedom mass-spring-dashpot system shown in Figure 9, where the output $y_{\mathrm{t}}$ represents the position of the second mass, and the control $u_{\mathrm{t}}$ represents the force applied to the second mass. The transfer function is $G_{0}(s)=\left(m_{1} s^{2}+c_{1} s+c_{2} s+k_{1}+k_{2}\right) /\left[\left(m_{1} s^{2}+c_{1} s+k_{1}\right)\left(m_{2} s^{2}+c_{2} s+k_{2}\right)+m_{2} c_{2} s^{3}+m_{2} k_{2} s^{2}\right]$, where $m_{1}=1, m_{2}=0.5, c_{1}=0.56 \pi, c_{2}=0.5 \pi, k_{1}=0.16 \pi^{2}$, and $k_{2}=0.5 \pi^{2}$.

The command $r_{\mathrm{t}}$ is an 80-second chirp with frequency content between 0.1 and $0.4 \mathrm{~Hz}$ as shown in Figure 10. The frequency range of $r_{\mathrm{t}}$ is selected to account for the bandwidth limitations of human motion. Humans cannot replicate arbitrarily high-frequency motion, and the $0.4 \mathrm{~Hz}$ upper limit is selected to be within the range of motion that a human can replicate. In addition, the frequency range is selected to include the natural frequencies associated with the zeros and dominant poles of $G_{0}$.

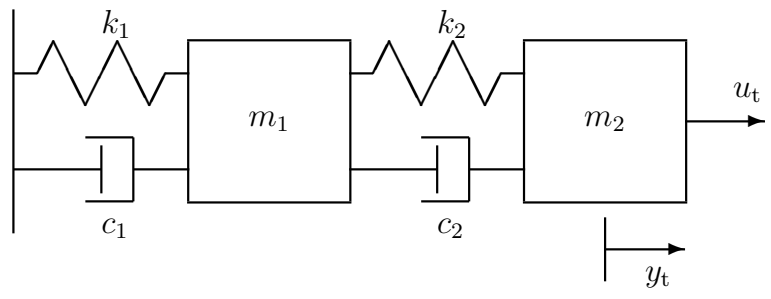

Figure 9: The two-degree-of-freedom mass-springdashpot system.

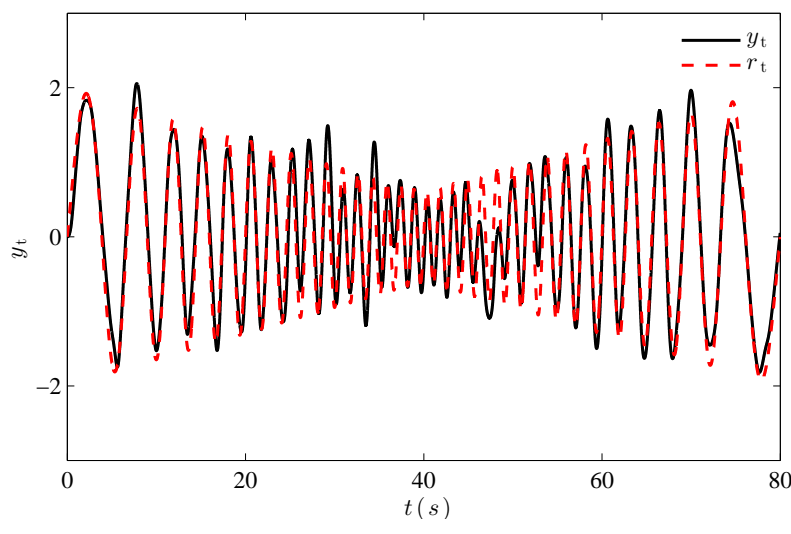

Figure 10: The reference $r_{\mathrm{t}}$ and output $y_{\mathrm{t}}$ for a single trial.

To demonstrate the SSID algorithm, we consider a single trial out of the 400 total trials (10 subjects and 40 trials per subject). In particular, we select the trial in which the time-averaged magnitude of the command-following error $\frac{1}{80} \int_{0}^{80}\left|e_{\mathrm{t}}(t)\right| \mathrm{d} t$ is minimized. The output $y_{\mathrm{t}}$ for this trial is shown in Figure 10. The subject's control strategy is modeled by $u(s)=G_{2}(s) e(s)+G_{1}(s) r(s)$, where $e, r, u$, and $y$ are the Laplace transforms of $e_{\mathrm{t}}, r_{\mathrm{t}}, u_{\mathrm{t}}$, and $y_{\mathrm{t}}$, respectively. The closed-loop transfer function from $r$ to $y$ is given by (1). The time domain signals $r_{\mathrm{t}}$ and $y_{\mathrm{t}}$ are divided into two segments of 40 seconds. For each pair of 
signal segments, we use the discrete Fourier transform to calculate frequency response data at the frequencies $\omega_{k}=2 \pi(0.1+0.025(k-1))$, where $k=1,2 \ldots, N=13$. The frequency response data from the two segments is averaged to obtain $\left\{H\left(\omega_{k}\right)\right\}_{k=1}^{N}$. We use Algorithm 1 and the data $\left\{H\left(\omega_{k}\right)\right\}_{k=1}^{N}$ to model the subject's the feedforward $G_{1}^{+}$and feedback $G_{2}^{+}$ controllers. We identify the best-fit second-order strictly proper feedback controller $G_{2}^{+}$and second-order improper feedforward controller $G_{1}^{+}$, where $n_{1}=4, n_{2}=1, d_{1}=2$, and $d_{2}=2$. The selected orders are sufficiently large to admit different control strategies that make $\left|e_{t}\right|$ small. For example, the selected orders allow for dynamic inversion in feedforward or high gain in feedback.

We choose a candidate pool such that the magnitude of poles of the feedforward controller, and the poles and zeros of the feedback controller are less than $25 \mathrm{rad} / \mathrm{s}$, because poles and zeros with magnitude greater than $25 \mathrm{rad} / \mathrm{s}$ have negligible effect on the Bode plot over the 0.1-to- $0.4 \mathrm{~Hz}$ frequency range of the chirp. The candidate pool is also selected such that the peak magnitude of the feedback controller is less than $30 \mathrm{~dB}$, which is an estimate of the peak gain that a human can use in feedback. Finally, we let $\rho=-0.1$. The candidate pool $\Phi$ contains approximately 160 billion elements and captures a wide range of second-order behavior over the frequency range of the chirp.

Algorithm 1 is implemented on the Lipscomb High Performance Computing Cluster at University of Kentucky. It takes approximately 3.5 hours to run Algorithm 1 using 32 Intel E5-2670@2.6Ghz cores.

Figure 11 shows the Bode plots of the identified transfer functions $G_{1}^{+}$and $G_{2}^{+}$, and the corresponding closed-loop transfer function $\tilde{\mathcal{G}}^{+}(s) \triangleq\left[G_{0}(s) G_{1}^{+}(s)+G_{0}(s) G_{2}^{+}(s)\right] /[1+$ $\left.G_{0}(s) G_{2}^{+}(s)\right]$. The identified feedforward controller $G_{1}^{+}$approximates $G_{0}^{-1}$ over the frequency range of $r_{\mathrm{t}}$, which suggests that this subject learned to control the system by using the inverse dynamics $G_{0}^{-1}$ in feedforward.
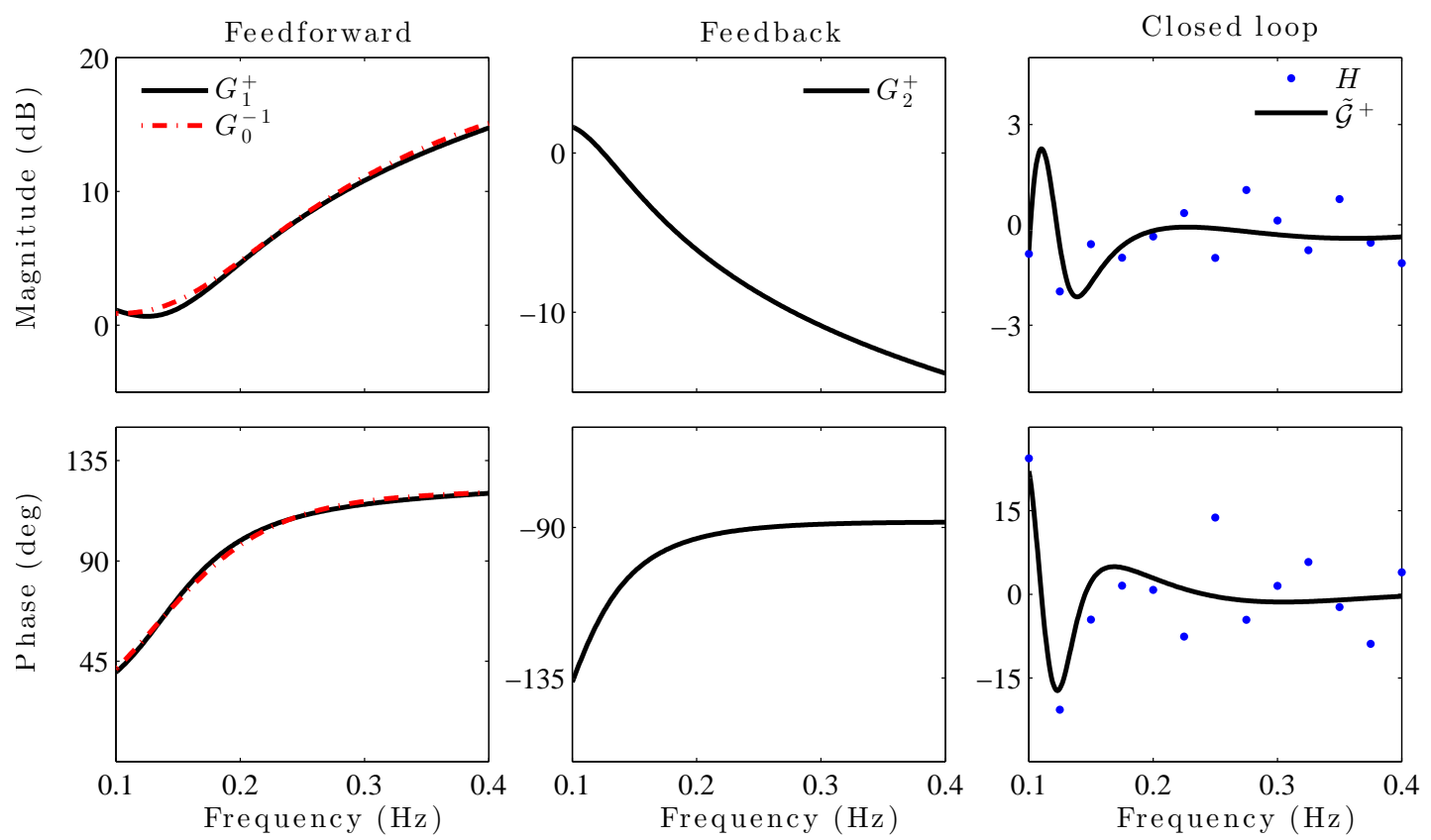

Figure 11: Bode plots of $G_{1}^{+}, G_{2}^{+}$, and $\tilde{\mathcal{G}}^{+}$for one trial with the minimized time-averaged magnitude of the command-following error. Note that $G_{1}^{+}$approximates $G_{0}^{-1}$. 


\section{Conclusion}

In this paper, we presented a frequency-domain SSID algorithm for identifying unknown feedback and feedforward subsystems interconnected with a known subsystem. This SSID method ensures asymptotic stability of the identified closed-loop transfer function. The method has application to modeling human control behavior (both feedback and feedforward). We demonstrated the algorithm on data from one trial of an experiment in which human subjects interacted with a dynamic system. For this trial, the identified feedforward controller approximated the inverse plant dynamics. In this case, the identified model of the control behavior agrees with the internal model hypothesis.

The main analytic results of the paper are Theorems 1-4, which describe the properties of the SSID algorithm. In particular, Theorem 4 shows that the coefficients of the identified feedback and feedforward transfer functions are arbitrarily close to the true coefficients if the candidate pool is sufficiently dense and the data noise is sufficiently small.

\section{Acknowledgements}

This work is supported by the National Science Foundation (award number: CMMI1405257) and the Kentucky Science and Engineering Foundation (award number: KSEF148-502-12-288). We also want to acknowledge Dr. Sheetz and Mr. Gazula from the Center for Computational Sciences at the University of Kentucky for providing assistance in coding Algorithm 1 for parallel processing.

\section{Appendix A. Proofs of Propositions 1 and 2}

Proof of Proposition 1. Let $\phi \in \mathcal{S}$. It follows from $(11)$ that $\Omega_{2}(\phi)$ is well defined and positive semidefinite. Next, assume for contradiction that $\Omega_{2}(\phi)$ is not positive definite. Thus, there exists a nonzero $x \in \mathbb{R}^{n_{1}+1}$ such that $x^{\mathrm{T}} \Omega_{2}(\phi) x=0$, and it follows from (11) that $0=x^{\mathrm{T}} \Omega_{2}(\phi) x=\sum_{k=1}^{N}\left|\frac{\tilde{\mathcal{N}}_{1}\left(\jmath \omega_{k}, \phi\right) x}{\tilde{\mathcal{D}}\left(\jmath \omega_{k}, \phi\right)}\right|^{2}$. Thus, for all $k \in \mathcal{N}, \tilde{\mathcal{N}}_{1}\left(\jmath \omega_{k}, \phi\right) x=0$. Next, it follows from (A3) that $N>d_{0}+d_{1}+d_{2}+n_{0}+\max \left\{n_{1}+d_{2}, n_{2}+d_{1}\right\} \geq d_{2}+n_{0}+n_{1}$. Since, $\tilde{\mathcal{N}}_{1}\left(\jmath \omega_{1}, \phi\right) x=\cdots=\tilde{\mathcal{N}}_{1}\left(\jmath \omega_{N}, \phi\right) x=0, \tilde{\mathcal{N}}_{1}(s, \phi) x$ is a degree $d_{2}+n_{0}+n_{1}$ polynomial, and $N>d_{2}+n_{0}+n_{1}$, it follows that $\tilde{\mathcal{N}}_{1}(s, \phi) x \equiv 0$. Moreover, since $\tilde{\mathcal{N}}_{1}(s, \phi) x \equiv 0, N_{0}(s) \not \equiv 0$ and $s^{d_{2}}+\Gamma_{d_{2}-1}^{\mathrm{T}}(s) E_{3} \phi \not \equiv 0$, it follows from (6) that $\Gamma_{n_{1}}^{\mathrm{T}}(s) x \equiv 0$. Thus, $x=0$, which is a contradiction. Therefore, $\Omega_{2}(\phi)$ is positive definite.

Proof of Proposition 2. Let $\phi \in \mathcal{S}$, and Proposition 1 implies that $\Omega_{2}(\phi)$ is positive definite. Define $x \triangleq-\frac{1}{2} \Omega_{2}^{-1}(\phi) \Omega_{1}(\phi) \in \mathbb{R}^{n_{1}+1}$, and let $\beta \in \mathbb{R}^{n_{1}+1}$ be such that $\beta \neq x$. Thus, (12) implies that

$$
J(\beta, \phi)=\beta^{\mathrm{T}} \Omega_{2}(\phi) \beta+\Omega_{1}^{\mathrm{T}}(\phi) \beta+\Omega_{0}(\phi)=[\beta-x]^{\mathrm{T}} \Omega_{2}(\phi)[\beta-x]+J(x, \phi) .
$$

Since $\Omega_{2}(\phi)$ is positive definite, it follows that $[\beta-x]^{\mathrm{T}} \Omega_{2}(\phi)[\beta-x]>0$, and (31) confirms the result.

\section{Appendix B. Proofs of Propositions 4 and 5}

Proof of Proposition 4. Since for all $k \in \mathcal{N}, \sigma\left(\jmath \omega_{k}\right)=0$, it follows that $H\left(\omega_{k}\right)=\tilde{G}\left(\jmath \omega_{k}\right)=$ $\tilde{\mathcal{G}}\left(\jmath \omega_{k}, \beta_{*}, \phi_{*}\right)$. Thus, it follows from (4) that for all $k \in \mathcal{N}$,

$$
\frac{\tilde{\mathcal{N}}_{2}\left(\jmath \omega_{k}, \phi_{*}\right)}{\tilde{\mathcal{D}}\left(\jmath \omega_{k}, \phi_{*}\right)}-H\left(\omega_{k}\right)=\frac{\tilde{\mathcal{N}}_{2}\left(\jmath \omega_{k}, \phi_{*}\right)}{\tilde{\mathcal{D}}\left(\jmath \omega_{k}, \phi_{*}\right)}-\tilde{\mathcal{G}}\left(\jmath \omega_{k}, \beta_{*}, \phi_{*}\right)=-\frac{\tilde{\mathcal{N}}_{1}\left(\jmath \omega_{k}, \phi_{*}\right) \beta_{*}}{\tilde{\mathcal{D}}\left(\jmath \omega_{k}, \phi_{*}\right)} .
$$

Next, substituting (32) into (10), and using (11) yields $\Omega_{1}\left(\phi_{*}\right)=-2 \Omega_{2}\left(\phi_{*}\right) \beta_{*}$. Since $\phi_{*} \in \mathcal{S}$, Proposition 1 implies that $\Omega_{2}\left(\phi_{*}\right)$ is positive definite, and thus, $\beta_{*}=-\frac{1}{2} \Omega_{2}^{-1}\left(\phi_{*}\right) \Omega_{1}\left(\phi_{*}\right)$. Moreover, (8) implies that $J\left(\beta_{*}, \phi_{*}\right)=\sum_{k=1}^{N} \underset{16}{\mid \tilde{G}}\left(\jmath \omega_{k}, \beta_{*}, \phi_{*}\right)-\left.H\left(\omega_{k}\right)\right|^{2}=0$. 
Proof of Proposition 5. Let $\beta \in \mathbb{R}^{n_{1}+1}$ and $\phi \in \mathcal{S}$. Since $\sum_{k=1}^{N}\left|\tilde{\mathcal{G}}\left(\jmath \omega_{k}, \beta, \phi\right)-\tilde{G}\left(\jmath \omega_{k}\right)\right|=0$, it follows that for all $k \in \mathcal{N}, \tilde{\mathcal{G}}\left(\jmath \omega_{k}, \beta, \phi\right)=\tilde{G}\left(\jmath \omega_{k}\right)$. Define $\mathcal{\mathcal { N }}(s) \triangleq \tilde{\mathcal{N}}_{1}(s, \phi) \beta+\tilde{\mathcal{N}}_{2}(s, \phi)$, $\tilde{\mathcal{N}}_{*}(s) \triangleq \tilde{\mathcal{N}}_{1}\left(s, \phi_{*}\right) \beta_{*}+\tilde{\mathcal{N}}_{2}\left(s, \phi_{*}\right)$, and $\psi(s) \triangleq \tilde{\mathcal{D}}\left(s, \phi_{*}\right) \tilde{\mathcal{N}}(s)-\tilde{\mathcal{D}}(s, \phi) \tilde{\mathcal{N}}_{*}(s)$. Since $\operatorname{deg} \tilde{\mathcal{N}} \leq n_{0}+$ $\max \left\{n_{1}+d_{2}, n_{2}+d_{1}\right\}, \operatorname{deg} \tilde{\mathcal{N}}_{*} \leq n_{0}+\max \left\{n_{1}+d_{2}, n_{2}+d_{1}\right\}$, and $\operatorname{deg} \tilde{\mathcal{D}}(s, \phi)=\operatorname{deg} \tilde{\mathcal{D}}\left(s, \phi_{*}\right)=$ $d_{0}+d_{1}+d_{2}$, it follows that $\operatorname{deg} \psi \leq d_{0}+d_{1}+d_{2}+n_{0}+\max \left\{n_{1}+d_{2}, n_{2}+d_{1}\right\}$. Since for all $k \in \mathcal{N}$, $\tilde{\mathcal{G}}\left(\jmath \omega_{k}, \beta, \phi\right)=\tilde{G}\left(\jmath \omega_{k}\right)$, it follows that for all $k \in \mathcal{N}, \psi\left(\jmath \omega_{k}\right)=0$. Next, it follows from (A3) that $\operatorname{deg} \psi<N$. Since for all $k \in \mathcal{N}, \psi\left(\jmath \omega_{k}\right)=0$, and $\operatorname{deg} \psi<N$, it follows that $\psi(s) \equiv 0$, which implies that $\tilde{\mathcal{D}}\left(s, \phi_{*}\right) \tilde{\mathcal{N}}(s) \equiv \tilde{\mathcal{D}}(s, \phi) \tilde{\mathcal{N}}_{*}(s)$. Thus, $\tilde{\mathcal{G}}(s, \beta, \phi) \equiv \tilde{\mathcal{G}}\left(s, \beta_{*}, \phi_{*}\right) \equiv \tilde{G}(s)$.

\section{Appendix C. Proofs of Propositions 6 and 9}

Proof of Proposition 6. Let $\phi \in \mathcal{S}$, and it follows that $\tilde{\mathcal{D}}(s, \phi)$ is Hurwitz. Thus, for all $k \in \mathcal{N}, \tilde{\mathcal{D}}\left(\jmath \omega_{k}, \phi\right) \neq 0$, and it follows from (9)-(11) that $\Omega_{0}, \Omega_{1}$, and $\Omega_{2}$ are well-defined and continuous on $\mathcal{S}$.

Next, it follows from Proposition 1 that $\Omega_{2}(\phi)$ is positive definite. Since $\Omega_{2}$ is continuous on $\mathcal{S}$, it follows that each element of $\Omega_{2}$ is continuous on $\mathcal{S}$, which implies that the adjugate of $\Omega_{2}$, denoted by adj $\Omega_{2}$, and the determinant of $\Omega_{2}$, denoted by $\operatorname{det} \Omega_{2}$, are continuous on $\mathcal{S}$. Since $\Omega_{2}$ is invertible on $\mathcal{S}$ and $\Omega_{2}^{-1}=\frac{1}{\operatorname{det} \Omega_{2}}$ adj $\Omega_{2}$, it follows that $\Omega_{2}^{-1}$ is continuous on $\mathcal{S}$.

Since $\Omega_{2}^{-1}$ and $\Omega_{1}$ are continuous on $\mathcal{S}$, it follows from (14) that $\theta$ is continuous on $\mathcal{S}$. Moreover, it follows from (12) that $J$ is continuous on $\mathbb{R}^{n_{1}+1} \times \mathcal{S}$. Since, in addition, $\theta$ is continuous on $\mathcal{S}$, it follows from (15) that $Q$ is continuous on $\mathcal{S}$.

Proof of Proposition 9. It follows (17) and (18) that $\hat{\Omega}_{0}$ and $\hat{\Omega}_{1}$ are continuous on $\mathcal{S} \times \mathbb{C}^{N}$. Also, it follows from $(11)$ that $\Omega_{2}$ is continuous on $\mathcal{S}$.

Next, it follows from Proposition 1 that $\Omega_{2}(\phi)$ is positive definite. Since $\Omega_{2}$ is continuous on $\mathcal{S}$, it follows that each element of $\Omega_{2}$ is continuous on $\mathcal{S}$, which implies that the adjugate of $\Omega_{2}$, denoted by adj $\Omega_{2}$, and the determinant of $\Omega_{2}$, denoted by $\operatorname{det} \Omega_{2}$, are continuous on $\mathcal{S}$. Since $\Omega_{2}$ is invertible on $\mathcal{S}$ and $\Omega_{2}^{-1}=\frac{1}{\operatorname{det} \Omega_{2}}$ adj $\Omega_{2}$, it follows that $\Omega_{2}^{-1}$ is continuous on $\mathcal{S}$.

Therefore, it follows from (20) and (22) that $\hat{J}$ is continuous on $\mathbb{R}^{n_{1}+1} \times \mathcal{S} \times \mathbb{C}^{N}$ and $\hat{\theta}$ is continuous on $\mathcal{S} \times \mathbb{C}^{N}$. Thus, it follows from $(21)$ that $\hat{Q}$ is continuous on $\mathcal{S} \times \mathbb{C}^{N}$.

\section{Appendix D. Proofs of Propositions 7 and 8}

The following notation is needed for the proofs of Propositions 7 and 8 . Let $x_{1}, \ldots, x_{n} \in$ $\mathbb{C}$, and let $x=<x_{1} \quad \ldots \quad x_{n}>$ be an unordered $n$-tuple of numbers in $\mathbb{C}$. See [35, Appendix V] for more details. Let $\mathbb{C}_{\text {sym }}^{n}$ denote the symmetric $n^{\text {th }}$ power of $\mathbb{C}$ as defined in [35, Appendix V]. Let $x=<x_{1} \quad \ldots \quad x_{n}>\in \mathbb{C}_{\text {sym }}^{n}$ and $y=<y_{1} \quad \ldots \quad y_{n}>\in \mathbb{C}_{\text {sym }}^{n}$, and define $\xi_{n}(x, y) \triangleq \min _{o} \max _{j=1, \ldots, n}\left|x_{j}-y_{o(j)}\right|$, where $o$ is any permutation of $1, \ldots, n$ and $o(j)$ denotes the image of $j$ through the permutation $o$. It follows from [35, Lemma 3D in Appendix V] that $\xi_{n}$ is a metric on $\mathbb{C}_{\text {sym }}^{n}$.

The following lemma is needed for the proofs of Propositions 7 and 8 .

Lemma 1. Consider $h_{n}: \mathbb{C}_{\text {sym }}^{n} \rightarrow \mathbb{C}^{n}$ given by

$$
h_{n}(x) \triangleq\left[\begin{array}{c}
-\sum_{j=1}^{n} x_{j} \\
\left(x_{1} x_{2}+\cdots+x_{1} x_{n}\right)+\left(x_{2} x_{3}+\cdots+x_{2} x_{n}\right)+\cdots+x_{n-1} x_{n} \\
\vdots \\
(-1)^{k} \sum_{1 \leq i_{1}<i_{2}<\cdots<i_{k} \leq n} \prod_{j=i_{1}}^{i_{k}} x_{j} \\
\vdots \\
(-1)^{n} \prod_{j=1}^{n} x_{j}
\end{array}\right]
$$


where $x=<x_{1} \quad \cdots \quad x_{n}>\in \mathbb{C}_{\mathrm{sym}}^{n}$. Consider the polynomial $P(s)=s^{n}+\tau_{1} s^{n-1}+\cdots+$ $\tau_{n-1} s+\tau_{n}$, where $\tau_{1}, \ldots, \tau_{n} \in \mathbb{C}$. Let $\lambda_{1}, \lambda_{2}, \ldots, \lambda_{n} \in \mathbb{C}$ denote the $n$ roots of $P$, and define $\tau \triangleq\left[\begin{array}{lll}\tau_{1} & \cdots & \tau_{n}\end{array}\right]^{\mathrm{T}} \in \mathbb{C}^{n}$ and $\lambda \triangleq<\lambda_{1} \quad \cdots \quad \lambda_{n}>\in \mathbb{C}_{\mathrm{sym}}^{n}$. Then, $h_{n}(\lambda)=\tau$, and $h_{n}$ and $h_{n}^{-1}$ are continuous.

Proof. It follows from [36, Fact 4.8.2] that $h_{n}(\lambda)=\tau$. Moreover, it follows from [35, Theorem 4A in Appendix V] that $h_{n}$ and $h_{n}^{-1}$ are continuous.

The following notation is needed for the proofs of Propositions 7 and 8 . Let $\kappa \triangleq d_{0}+d_{2}$. Let $h_{d_{1}}: \mathbb{C}_{\text {sym }}^{d_{1}} \rightarrow \mathbb{C}^{d_{1}}$ and $h_{\kappa}: \mathbb{C}_{\text {sym }}^{\kappa} \rightarrow \mathbb{C}^{\kappa}$ be functions given by (33), where $n$ is replaced by $d_{1}$ and $\kappa$, respectively. Let $a \triangleq\left[\begin{array}{llll}a_{1} & a_{2} & \cdots & a_{d_{0}}\end{array}\right]^{\mathrm{T}} \in \mathbb{R}^{d_{0}}$ and $b \triangleq\left[\begin{array}{llll}b_{0} & b_{1} & \cdots & b_{n_{0}}\end{array}\right]^{\mathrm{T}} \in$ $\mathbb{R}^{n_{0}+1}$ be such that $N_{0}(s)=\Gamma_{n_{0}}^{\mathrm{T}}(s) b$ and $D_{0}(s)=s^{d_{0}}+\Gamma_{d_{0}-1}^{\mathrm{T}}(s) a$. Consider the continuous function $g: \mathbb{R}^{d-d_{1}} \rightarrow \mathbb{R}^{\kappa}$ given by

$$
g(z) \triangleq A z+B
$$

where $B \triangleq\left[\begin{array}{ll}a^{\mathrm{T}} & 0_{1 \times d_{2}}\end{array}\right]^{\mathrm{T}} \in \mathbb{R}^{\kappa}$ and

$$
A \triangleq\left[\begin{array}{cccc:cccc}
0 & 0 \\
\hdashline\left(\kappa-n_{0}-n_{2}-1\right) \times\left(n_{2}+1\right) & 1 & 0 & \cdots & 0 \\
b_{0} & 0 & \cdots & 0 & a_{1} & 1 & \ddots & \vdots \\
b_{1} & b_{0} & \ddots & \vdots & a_{2} & a_{1} & \ddots & 0 \\
\vdots & b_{1} & \ddots & 0 & \vdots & a_{2} & \ddots & 1 \\
b_{n_{0}} & \vdots & \ddots & b_{0} & a_{d_{0}} & \vdots & \ddots & a_{1} \\
0 & b_{n_{0}} & & b_{1} & 0 & a_{d_{0}} & & a_{2} \\
\vdots & & \ddots & \vdots & \vdots & & \ddots & \vdots \\
0 & 0 & & b_{n_{0}} & 0 & 0 & & a_{d_{0}}
\end{array}\right] \in \mathbb{R}^{\kappa \times\left(d-d_{1}\right)} .
$$

Proof of Proposition \%. Since $\Psi$ is compact, it follows that $\Psi$ is bounded, which implies that $\left(\Psi \cap \mathcal{S}_{\rho}\right) \subseteq \Psi$ is bounded.

Let $\phi \in\left(\Psi \cap \mathcal{S}_{\rho}\right)$, and we show that $\phi$ is not an isolated point. Let $\phi=\left[\begin{array}{ll}x^{\mathrm{T}} & z^{\mathrm{T}}\end{array}\right]^{\mathrm{T}}$, where $x \in \mathbb{R}^{d_{1}}$ and $z \in \mathbb{R}^{d^{-d_{1}}}$. Since $\phi \in \Psi$ and $\Psi$ is perfect, it follows that there exists a sequence $\left\{\phi_{i}\right\}_{i=1}^{\infty} \subseteq \Psi$ that converges to $\phi$. For each $i \in \mathbb{Z}^{+}$, let $\phi_{i}=\left[\begin{array}{ll}x_{i}^{\mathrm{T}} & z_{i}^{\mathrm{T}}\end{array}\right]^{\mathrm{T}}$, where $x_{i} \in \mathbb{R}^{d_{1}}$ and $z_{i} \in \mathbb{R}^{d-d_{1}}$. Thus, $\left\{x_{i}\right\}_{i=1}^{\infty}$ converges to $x$ and $\left\{z_{i}\right\}_{i=1}^{\infty}$ converges to $z$.

Let $\mu_{1}, \ldots, \mu_{d_{1}} \in \mathbb{C}$ denote the $d_{1}$ roots of $\mathcal{D}_{1}(s, \phi)$, and define $p \triangleq<\mu_{1} \ldots \mu_{d_{1}}>$. For each $i \in \mathbb{Z}^{+}$, let $\mu_{i, 1}, \ldots, \mu_{i, d_{1}} \in \mathbb{C}$ denote the $d_{1}$ roots of $\mathcal{D}_{1}\left(s, \phi_{i}\right)$, and define $p_{i} \triangleq<$

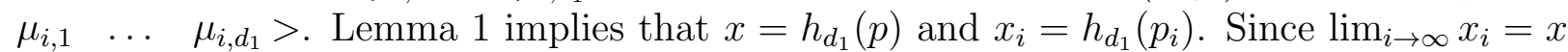
and Lemma 1 implies that $h_{d_{1}}^{-1}$ is continuous, it follows that $\lim _{i \rightarrow \infty} p_{i}=\lim _{i \rightarrow \infty} h_{d_{1}}^{-1}\left(x_{i}\right)=$ $h_{d_{1}}^{-1}(x)=p$. Since $\phi \in \mathcal{S}_{\rho}$, it follows that for all $j=1, \ldots, d_{1}$, Re $\mu_{j}<\rho$. Define $\epsilon_{1} \triangleq$ $\min _{j=1, \ldots, d_{1}} \rho-\operatorname{Re} \mu_{j}$. Since $\lim _{i \rightarrow \infty} p_{i}=p$ and $\xi_{d_{1}}$ is a metric on $\mathbb{C}_{\mathrm{sym}}^{d_{1}}$, it follows that there exists $L_{1} \in \mathbb{Z}^{+}$such that for all $i \geq L_{1}, \xi_{d_{1}}\left(p_{i}, p\right)<\epsilon_{1}$. Thus, for each $i \geq L_{1}$, there exists a permutation $o_{i}$ of $1, \ldots, d_{1}$ such that for all $j=1, \ldots, d_{1},\left|\mu_{i, j}-\mu_{o_{i}(j)}\right|<\epsilon_{1}$. Therefore, for all $i \geq L_{1}$ and all $j=1, \ldots, d_{1}$,

$$
\operatorname{Re} \mu_{i, j}-\operatorname{Re} \mu_{o_{i}(j)} \leq\left|\operatorname{Re} \mu_{i, j}-\operatorname{Re} \mu_{o_{i}(j)}\right| \leq\left|\mu_{i, j}-\mu_{o_{i}(j)}\right|<\epsilon_{1} \leq \rho-\operatorname{Re} \mu_{o_{i}(j)},
$$

which implies that $\operatorname{Re} \mu_{i, j}<\rho$.

Next, since (A2) implies that $\kappa>n_{0}+n_{2}$, it follows that $D_{0}(s) \mathcal{D}_{2}(s, \phi)+N_{0}(s) \mathcal{N}_{2}(s, \phi)$ is a monic degree $\kappa$ polynomial, which can be written as $s^{\kappa}+\Gamma_{\kappa-1}^{\mathrm{T}}(s) \gamma$, where $\gamma \in \mathbb{R}^{\kappa}$. Let $\nu_{1}, \ldots, \nu_{\kappa} \in \mathbb{C}$ denote the $\kappa$ roots of $D_{0}(s) \mathcal{D}_{2}(s, \phi)+N_{0}(s) \mathcal{N}_{2}(s, \phi)$. For each $i \in \mathbb{Z}^{+}$, let $\nu_{i, 1}, \ldots, \nu_{i, \kappa}$ denote the $\kappa$ roots of $D_{0}(s) \mathcal{D}_{2}\left(s, \phi_{i}\right)+N_{0}(s) \mathcal{N}_{2}\left(s, \phi_{i}\right)$, which can be written as $s^{\kappa}+\Gamma_{\kappa-1}^{\mathrm{T}}(s) \gamma_{i}$, where $\gamma_{i} \in \mathbb{R}^{\kappa}$. Using (34), it follows that $\gamma=g(z)$ and $\gamma_{i}=g\left(z_{i}\right)$. Define 
$q \triangleq<\nu_{1} \quad \cdots \quad \nu_{\kappa}>$ and $q_{i} \triangleq<\nu_{i, 1} \quad \cdots \quad \nu_{i, \kappa}>$, and Lemma 1 implies that $\gamma=g(z)=h_{\kappa}(q)$ and $\gamma_{i}=g\left(z_{i}\right)=h_{\kappa}\left(q_{i}\right)$. Since $\lim _{i \rightarrow \infty} z_{i}=z$ and $g$ is continuous on $\mathbb{R}^{d-d_{1}}$, it follows that $\lim _{i \rightarrow \infty} \gamma_{i}=\lim _{i \rightarrow \infty} g\left(z_{i}\right)=g(z)=\gamma$. Since $\lim _{i \rightarrow \infty} \gamma_{i}=\gamma$ and Lemma 1 implies that $h_{\kappa}^{-1}$ is continuous, it follows that $\lim _{i \rightarrow \infty} q_{i}=\lim _{i \rightarrow \infty} h_{\kappa}^{-1}\left(\gamma_{i}\right)=h_{\kappa}^{-1}(\gamma)=q$. Since $\phi \in \mathcal{S}_{\rho}$, it follows that for all $l=1, \ldots, \kappa$, Re $\nu_{l}<\rho$. Define $\epsilon_{2} \triangleq \min _{l=1, \ldots, \kappa} \rho-\operatorname{Re} \nu_{l}$. Since $\lim _{i \rightarrow \infty} q_{i}=q$ and $\xi_{\kappa}$ is a metric on $\mathbb{C}_{\text {sym }}^{\kappa}$, it follows that there exists $L_{2} \in \mathbb{Z}^{+}$such that for all $i \geq L_{2}$, $\xi_{\kappa}\left(q_{i}, q\right)<\epsilon_{2}$. Thus, for each $i \geq L_{2}$, there exists a permutation $o_{i}$ of $1, \ldots, \kappa$ such that for all $l=1, \ldots, \kappa,\left|\nu_{i, l}-\nu_{o_{i}(l)}\right|<\epsilon_{2}$. Therefore, for all $i \geq L_{2}$ and all $l=1, \ldots, \kappa$,

$\operatorname{Re} \nu_{i, l}-\operatorname{Re} \nu_{o_{i}(l)} \leq\left|\operatorname{Re} \nu_{i, l}-\operatorname{Re} \nu_{o_{i}(l)}\right| \leq\left|\nu_{i, l}-\nu_{o_{i}(l)}\right|<\epsilon_{2} \leq \rho-\operatorname{Re} \nu_{o_{i}(l)}$,

which implies that $\operatorname{Re} \nu_{i, l}<\rho$.

Define $L \triangleq \max \left\{L_{1}, L_{2}\right\}$. Let $i \geq L$, and (5) implies that $\mu_{i, 1}, \ldots, \mu_{i, d_{1}}$ and $\nu_{i, 1}, \ldots, \nu_{i, \kappa}$ are the $d_{0}+d_{1}+d_{2}$ roots of $\tilde{\mathcal{D}}\left(s, \phi_{i}\right)$. For all $j=1, \ldots, d_{1}$ and $l=1, \ldots, \kappa, \operatorname{Re} \mu_{i, j}<\rho$ and $\operatorname{Re} \nu_{i, l}<\rho$, which implies that $\tilde{\mathcal{D}}\left(s+\rho, \phi_{i}\right)$ is Hurwitz. Thus, $\phi_{i} \in \mathcal{S}_{\rho}$, which implies that $\phi_{i} \in\left(\Psi \cap \mathcal{S}_{\rho}\right)$. Therefore, $\left\{\phi_{i}\right\}_{i=L}^{\infty} \subseteq\left(\Psi \cap \mathcal{S}_{\rho}\right)$ converges to $\phi \in\left(\Psi \cap \mathcal{S}_{\rho}\right)$, which implies that $\phi$ is not an isolated point.

Proof of Proposition 8. Since $\Psi$ is compact, it follows that $\overline{\Psi \cap \mathcal{S}_{\rho}} \subseteq\left(\bar{\Psi} \cap \overline{\mathcal{S}}_{\rho}\right)=\Psi \cap \overline{\mathcal{S}_{\rho}}$, which implies that $\overline{\Psi \cap \mathcal{S}_{\rho}}$ is compact.

We now show that $\frac{\rho}{\Psi \cap \mathcal{S}_{\rho}} \subseteq \mathcal{S}$. Define $E_{4} \triangleq\left[\begin{array}{cc}E_{2}^{\mathrm{T}} & E_{3}^{\mathrm{T}}\end{array}\right]^{\mathrm{T}} \in \mathbb{R}^{\left(d-d_{1}\right) \times d}, \mathcal{F} \triangleq\{\lambda \in$ $\mathbb{C}: \operatorname{Re} \lambda \leq \rho<0\}$, and

$S_{0} \triangleq\left\{\phi \in \mathbb{R}^{d}:\right.$ if $\lambda \in \mathbb{C}$ and $\tilde{\mathcal{D}}(\lambda, \phi)=0$, then $\left.\lambda \in \mathcal{F}\right\}$,

$S_{1} \triangleq\left\{x \in \mathbb{R}^{d_{1}}: x=E_{1} \phi\right.$, where $\phi \in \mathbb{R}^{d}$ and if $\lambda \in \mathbb{C}$ and $\mathcal{D}_{1}(\lambda, \phi)=0$, then $\left.\lambda \in \mathcal{F}\right\}$,

$S_{2} \triangleq\left\{z \in \mathbb{R}^{d-d_{1}}: z=E_{4} \phi\right.$, where $\phi \in \mathbb{R}^{d}$ and if $\lambda \in \mathbb{C}$ and

$$
\left.D_{0}(\lambda) \mathcal{D}_{2}(\lambda, \phi)+N_{0}(\lambda) \mathcal{N}_{2}(\lambda, \phi)=0 \text {, then } \lambda \in \mathcal{F}\right\} \text {. }
$$

Let $x \in S_{1}, z \in S_{2}$, and $\phi=\left[\begin{array}{ll}x^{\mathrm{T}} & z^{\mathrm{T}}\end{array}\right]^{\mathrm{T}} \in S_{0}$.

First, we show that $S_{1}$ is closed in $\mathbb{R}^{d_{1}}$. Let $\mu_{1}, \ldots, \mu_{d_{1}}$ denote the $d_{1}$ roots of $\mathcal{D}_{1}(s, \phi)$, and define $p \triangleq<\mu_{1}, \cdots, \quad \mu_{d_{1}}>$. Since $x \in S_{1}$, it follows that $\mu_{1}, \ldots, \mu_{d_{1}} \in \mathcal{F}$ and $\mu \in \mathcal{F}_{\text {sym }}^{d_{1}}$. Lemma 1 implies that $x=h_{d_{1}}(p) \in h_{d_{1}}\left(\mathcal{F}_{\text {sym }}^{d_{1}}\right)$, which implies that $x \in\left(\mathbb{R}^{d_{1}} \cap h_{d_{1}}\left(\mathcal{F}_{\text {sym }}^{d_{1}}\right)\right)$. Thus, $S_{1} \subseteq\left(\mathbb{R}^{d_{1}} \cap h_{d_{1}}\left(\mathcal{F}_{\text {sym }}^{d_{1}}\right)\right)$. Next, let $\hat{x} \in\left(\mathbb{R}^{d_{1}} \cap h_{d_{1}}\left(\mathcal{F}_{\text {sym }}^{d_{1}}\right)\right)$, and let $\hat{\mu}_{1}, \ldots, \hat{\mu}_{d_{1}}$ denote the $d_{1}$ roots of $\mathcal{D}_{1}\left(s,\left[\begin{array}{cc}\hat{x}^{\mathrm{T}} & z^{\mathrm{T}}\end{array}\right]^{\mathrm{T}}\right)$. Thus, $\hat{x} \in \mathbb{R}^{d_{1}}$ and $h_{d_{1}}^{-1}(\hat{x}) \in \mathcal{F}_{\text {sym }}^{d_{1}}$, which implies that $\hat{\mu}_{1}, \ldots, \hat{\mu}_{d_{1}} \in \mathcal{F}$. Thus, $\hat{x} \in S_{1}$, which implies that $\left(\mathbb{R}^{d_{1}} \cap h_{d_{1}}\left(\mathcal{F}_{\text {sym }}^{d_{1}}\right)\right) \subseteq S_{1}$. Therefore, $S_{1}=\mathbb{R}^{d_{1}} \cap h_{d_{1}}\left(\mathcal{F}_{\text {sym }}^{d_{1}}\right)$. Since $\mathcal{F}$ is closed in $\mathbb{C}$, it follows that $\mathcal{F}_{\text {sym }}^{d_{1}}$ is closed in $\mathbb{C}_{\text {sym }}^{d_{1}}$. Since Lemma 1 implies that $h_{d_{1}}^{-1}$ is continuous, it follows that $h_{d_{1}}\left(\mathcal{F}_{\text {sym }}^{d_{1}}\right)$ is closed in $\mathbb{C}^{d_{1}}$. Thus, $S_{1}=\mathbb{R}^{d_{1}} \cap h_{d_{1}}\left(\mathcal{F}_{\text {sym }}^{d_{1}}\right)$ is closed in $\mathbb{R}^{d_{1}}$.

Next, we show that $S_{2}$ is closed in $\mathbb{R}^{d-d_{1}}$. Since (A2) implies that $\kappa>n_{0}+n_{2}$, it follows that $D_{0}(s) \mathcal{D}_{2}(s, \phi)+N_{0}(s) \mathcal{N}_{2}(s, \phi)$ is a monic degree $\kappa$ polynomial, which can be written as $s^{\kappa}+\Gamma_{\kappa-1}^{\mathrm{T}}(s) \gamma$, where $\gamma \in \mathbb{R}^{\kappa}$. Using (34), it follows that $\gamma=g(z)$. Let $\nu_{1}, \ldots, \nu_{\kappa}$ denote the $\kappa$ roots of $s^{\kappa}+\Gamma_{\kappa-1}^{\mathrm{T}}(s) \gamma$, and define $q \triangleq\left\langle\nu_{1}, \cdots, \nu_{\kappa}>\right.$. Since $z \in S_{2}$, it follows that $\nu_{1}, \ldots, \nu_{\kappa} \in \mathcal{F}$ and $q \in \mathcal{F}_{\text {sym }}^{\kappa}$. Lemma 1 implies that $\gamma=h_{\kappa}(q) \in h_{\kappa}\left(\mathcal{F}_{\text {sym }}^{\kappa}\right)$, which implies that $z=g^{-1}(\gamma) \in g^{-1}\left(\mathbb{R}^{\kappa} \cap h_{\kappa}\left(\mathcal{F}_{\text {sym }}^{\kappa}\right)\right)$. Thus, $S_{2} \subseteq g^{-1}\left(\mathbb{R}^{\kappa} \cap h_{\kappa}\left(\mathcal{F}_{\text {sym }}^{\kappa}\right)\right)$. Next, let $\hat{z} \in g^{-1}\left(\mathbb{R}^{\kappa} \cap h_{\kappa}\left(\mathcal{F}_{\text {sym }}^{\kappa}\right)\right) \subseteq \mathbb{R}^{d-d_{1}}$. Thus, $g(\hat{z}) \in\left(\mathbb{R}^{\kappa} \cap h_{\kappa}\left(\mathcal{F}_{\text {sym }}^{\kappa}\right)\right)$. Let $\hat{\nu}_{1}, \ldots, \hat{\nu}_{\kappa}$ denote the $\kappa$ roots of $s^{\kappa}+\Gamma_{\kappa-1}^{\mathrm{T}}(s) g(\hat{z})$. It follows from Lemma 1 that $\left\langle\hat{\nu}_{1}, \quad \cdots, \quad \hat{\nu}_{\kappa}>=h_{\kappa}^{-1}(g(\hat{z})) \in \mathcal{F}_{\mathrm{sym}}^{\kappa}\right.$, which implies that $\hat{\nu}_{1}, \ldots, \hat{\nu}_{\kappa} \in \mathcal{F}$. Thus, $\hat{z} \in S_{2}$, which implies that $g^{-1}\left(\mathbb{R}^{\kappa} \cap h_{\kappa}\left(\mathcal{F}_{\text {sym }}^{\kappa}\right)\right) \subseteq S_{2}$. Therefore, $S_{2}=g^{-1}\left(\mathbb{R}^{\kappa} \cap h_{\kappa}\left(\mathcal{F}_{\text {sym }}^{\kappa}\right)\right)$. Since $\mathcal{F}$ is closed in $\mathbb{C}$, it follows that $\mathcal{F}_{\text {sym }}^{\kappa}$ is closed in $\mathbb{C}_{\text {sym }}^{\kappa}$. Since Lemma 1 implies that $h_{\kappa}^{-1}$ is continuous, it follows that $h_{\kappa}\left(\mathcal{F}_{\text {sym }}^{\kappa}\right)$ is closed in $\mathbb{C}^{\kappa}$. Thus, $\mathbb{R}^{\kappa} \cap h_{\kappa}\left(\mathcal{F}_{\text {sym }}^{\kappa}\right)$ is closed in $\mathbb{R}^{\kappa}$. Since, in addition, $g$ is continuous, it follows that $S_{2}=g^{-1}\left(\mathbb{R}^{\kappa} \cap h_{\kappa}\left(\mathcal{F}_{\text {sym }}^{\kappa}\right)\right)$ is closed in $\mathbb{R}^{d-d_{1}}$. 
Since $S_{1}$ is closed in $\mathbb{R}^{d_{1}}, S_{2}$ is closed in $\mathbb{R}^{d-d_{1}}$, and (5) implies that $S_{0}=S_{1} \times S_{2}$, it follows that $S_{0}$ is closed in $\mathbb{R}^{d}$. Since, in addition, $\mathcal{S}_{\rho} \subseteq S_{0}$, it follows that $\overline{\mathcal{S}}_{\rho} \subseteq S_{0}$. Thus, $\overline{\Psi \cap \mathcal{S}_{\rho}} \subseteq\left(\Psi \cap \overline{\mathcal{S}}_{\rho}\right) \subseteq \overline{\mathcal{S}}_{\rho} \subseteq S_{0} \subseteq \mathcal{S}$

\section{References}

[1] M. Kawato, Internal models for motor control and trajectory planning, Current Opinion in Neurobiology 9 (1999) 718-727.

[2] D. M. Wolpert, R. C. Miall, M. Kawato, Internal models in the cerebellum, Trends in Cognitive Sciences 2 (1998) 338-347.

[3] J. R. Flanagan, A. M. Wing, Modulation of grip force with load force during point-to-point movements, Experimental Brain Research 95 (1993) 131-143.

[4] J. R. Flanagan, J. R. Tresilian, A. M. Wing, Coupling of grip force and load force during arm movements with grasped objects, Neuroscience Letters 152 (1993) 53-56.

[5] J. R. Flanagan, J. R. Tresilian, Grip-load force coupling: A general control strategy for transporting objects, Journal of Experimental Psychology: Human Perception and Performance 20 (1994) 944-957.

[6] R. Shadmehr, F. A. Mussa-Ivaldi, Adaptive representation of dynamics during learning of a motor task, The Journal of Neuroscience 14 (5) (1994) 3208-3224.

[7] D. M. Wolpert, Z. Ghahramani, M. I. Jordan, An internal model for sensorimotor integration, Science 269 (1995) 1880-1882.

[8] D. A. Nowak, J. Hermsdorfer, S. Glasauer, L. Meyer, N. Mai, The effects of digital anaesthesia on predictive grip force adjustments during vertical movements of a grasped object, European Journal of Neuroscience 14 (2001) 756-762.

[9] D. A. Nowak, S. Glasauer, L. Meyer, N. Mai, J. Hermsdorfer, The role of cutaneous feedback for anticipatory grip force adjustments during object movements and externally imposed variation of the direction of gravity, Somatosensory and Motor Research 19 (2002) 49-60.

[10] D. W. Franklin, R. Osu, E. Burdet, M. Kawato, T. E. Milner, Adaptation to stable and unstable dynamics achieved by combined impedance control and inverse dynamics model, Journal of neurophysiology 90 (5) (2003) 3270-3282.

[11] D. J. Ostry, A. G. Feldman, A critical evaluation of the force control hypothesis in motor control, Experimental Brain Research 221 (2003) 275-288.

[12] M. Roth, J. Leasage, L. Litz, Block-box identification of discrete event systems with optimal partitioning of concurrent subsystems, in: Proc. Amer. Contr. Conf., Baltimore, MD, 2010, pp. 2601-2606.

[13] M. Suzuki, N. Takatsuki, J. Imura, K. Aihara, Node knock-out based structure identification in networks of identical multi-dimensional subsystems, in: Proc. Euro. Contr. Conf., Zurich, Switzerland, 2013, pp. 2280-2285.

[14] J. C. Spall, Identification for systems with binary subsystems, IEEE Trans. Auto. Contr. 59 (2014) $3-17$.

[15] S. Prakriya, D. Hatzinakos, Blind identification of linear subsystems of LTI-ZMNL-LTI models with cyclostationary inputs, IEEE Trans. Sig. Proc. 45 (1997) 2023-2036.

[16] A. Fagergren, O. Ekeberg, H. Forssberg, Precision grip force dynamics: A system identification approach, IEEE Trans. Biomedical Engineering 47 (2000) 1366-1375.

[17] W. Zhao, H. Chen, Recursive identification for Hammerstein system with ARX subsystem, in: Proc. Chin. Contr. Conf., Harbin, China, 2006, pp. 473-476.

[18] D. Schmid, G. Enzner, Robust subsystems for iterative multichannel blind system identification and equalization, in: Proc. Pacific Rim Conf. on Communications, Computers and Signal Processing, Victoria, Canada, 2009, pp. 889-893.

[19] A. Ghoreyshi, H. L. Galiana, Simultaneous identification of oculomotor subsystems using a hybrid system approach: Introducing hybrid extended least squares, IEEE Trans. Biomedical Engineering 57 (2010) 1089-1098.

[20] S. Hatakeyama, M. Iwase, S. Yamaura, Analysis of human operation utilizing closed-loop identification method, in: Proc. IEEE Workshop on Intelligent Data Acquisition and Advanced Computing System: Technology and Applications, Sofia, Bulgaria, 2005, pp. 232-236.

[21] S. Gillijns, B. D. Moor, Data-based subsystem identification for dynamic model updating, in: Proc. Conf. Dec. Contr., San Diego, CA, 2006, pp. 3303-3308.

[22] H. J. Palanthandalam-Madapusi, S. Gillijns, B. D. Moor, D. S. Bernstein, Subsystem identification for nonlinear model updating, in: Proc. Amer. Contr. Conf., Minneapolis, MN, 2006, pp. 3056-3061.

[23] A. V. Morozov, A. A. Ali, A. M. D'Amato, A. J. Ridley, S. L. Kukreja, D. S. Bernstein, Retrospectivecost-based model refinement for system emulation and subsystem identification, in: Proc. Conf. Dec. 
Contr., Orlando, FL, 2011, pp. 2142-2147.

[24] A. M. D'Amato, A. J. Ridley, D. S. Bernstein, Retrospective-cost-based adaptive model refinement for the ionosphere and thermosphere, Statistical Analysis and Data Mining 4 (2011) 446-458.

[25] D. T. McRuer, E. S. Krendel, The human control operator as a servo system element, Journal of the Franklin Institute 6 (267) (1959) 511-536.

[26] D. T. McRuer, D. Graham, E. S. Krendel, Manual control of a single-loop system: Part i, Journal of the Franklin Institute 1 (283) (1967) 1-29.

[27] D. T. McRuer, D. Graham, E. S. Krendel, Manual control of a single-loop system: Part ii, Journal of the Franklin Institute 2 (283) (1967) 145-168.

[28] H. van der Kooij, E. de Vlugt, Postural responses evoked by platform pertubations are dominated by continuous feedback, Journal of Neurophysiology 98 (2) (2007) 730-743.

[29] T. Kiemel, Y. Zhang, J. J. Jeka, Identification of neural feedback for upright stance in humans: Stabilizartion rather than sway minimization, Journal of Neuroscience 31 (42) (2011) 15144-15153.

[30] F. M. Drop, D. M. Pool, H. J. Damveld, M. M. van Paassen, M. Mulder, Identification of the feedforward component in manual control with predictable target signals, IEEE Trans. Cybernetics 43 (6) (2013) 1936-1949.

[31] V. A. Laurense, D. M. Pool, H. J. Damveld, M. M. van Paassen, M. Mulder, Effect of controlled element dynamics on human feedforward behavior in ramp-tracking tasks, IEEE Trans. Cybernetics 45 (2) (2015) 253-265.

[32] X. Zhang, S. Wang, T. M. Seigler, J. B. Hoagg, A subsystem identification technique for modeling control strategies used by humans, in: Proc. Amer. Contr. Conf., Portland, OR, 2014, pp. 2827-2832.

[33] R. Beals, Analysis An Introduction, Cambridge University Press, Cambridge, 2004.

[34] R. K. Sundaram, A First Course in Optimization Theory, Cambridge University Press, Cambridge, 1996.

[35] H. Whitney, Complex Analytic Varieties, Addison-Wesley Publishing Company, 1972.

[36] D. S. Bernstein, Matrix Mathematics, 2nd Edition, Princeton University Press, 2009. 